\begin{tabular}{|c|l|}
\hline Title & Dynamic boundary conditions for Hamilton-Jacobi equations \\
\hline Author(s) & Elliott, C. M; Giga, Y.; Goto, S. \\
\hline Citation & Hokkaido University Preprint Series in Mathematics, 546, 1-27 \\
\hline Issue Date & 2001-12 \\
\hline DOI & 10.14943/83691 \\
\hline Doc URL & http://hdl.handle.net/2115/69295 \\
\hline Type & bulletin (article) \\
\hline File Information & pre546.pdf \\
\hline
\end{tabular}

Instructions for use 
Dynamic boundary conditions for Hamilton-Jacobi equations

C. M. Elliott, Y. Giga, S. Goto

Series \#546. December 2001 


\section{HOKKAIDO UNIVERSITY PREPRINT SERIES IN MATHEMATICS}

\#519 Y. Giga, Viscosity solutions with shocks, 58 pages. 2001.

\#520 A. Inoue, On the worst conditional expectation, 10 pages. 2001.

\#521 Yumiharu Nakano, Efficient hedging with coherent risk measure, 10 pages. 2001.

\#522 T. Nakazi, Toeplitz operators and weighted norm inequalities on the bidisc, 15 pages. 2001.

\#523 T. Mikami, Covariance kernel and the central limit theorem in the total variation distance, 80 pages. 2001.

\#524 K. Yamaguchi and T. Yatsui, Geometry of higher order differential equations of finite type associated with symmetric spaces, 43 pages. 2001.

\#525 T. Suwa, Residues of Chern classes, 20 pages. 2001.

\#526 V. Anh and A. Inoue, Dynamic models of asset prices with long memory, 21 pages. 2001.

\#527 T. Izawa and T. Suwa, Multiplicity of functions on singular varieties, 21 pages. 2001.

\#528 T. Nakazi and T. Yamamoto, Two dimensional commutative Banach algebras and von Neumann inequality, 18 pages. 2001.

\#529 Y. Giga, N. Ishimura and Y. Kohsaka, Spiral solutions for a weakly anisotropic curvature flow equation, 16 pages. 2001.

\#530 Y. Giga and P. Rybka, Quasi-static evolution of 3-D crystals grown from supersaturated vapor, 16 pages. 2001.

\#531 Y. Tonegawa, Remarks on convergence of the Allen-Cahn equation, 18 pages. 2001.

\#532 T. Suwa, Characteristic classes of singular varieties, 26 pages. 2001.

\#533 J. Escher, Y. Giga and K. Ito, On a limiting motion and self-intersections for the intermediate surface diffusion flow, 20 pages. 2001.

\#534 Y.-H. R. Tsai, Y. Giga and S. Osher, A level set approach for computing discontinuous solutions of a class of Hamilton-Jacobi equations, 30 pages. 2001.

\#535 A. Yamagami, On Gouvêa's conjecture in the unobstructed case, 19 pages. 2001.

\#536 A. Inoue, What does the partial autocorrelation function look like for large lags, 27 pages. 2001.

\#537 T. Nakazi and T. Yamamoto, Norm of a linear combination of two operators of a Hilbert space, 16 pages. 2001.

\#538 Y. Giga, On the two-dimensional nonstationary vorticity equations, 12 pages. 2001.

\#539 M. Jinzenji, Gauss-Manin system and the virtual structure constants, 25 pages. 2001.

\#540 H. Ishii and T.Mikami, Motion of a graph by $R$-curvature, 28 pages. 2001.

\#541 M. Jinzenji and T. Sasaki, $\mathcal{N}=4$ supersymmetric Yang-Mills theory on orbifold $-T^{4} / \mathbf{Z}_{2}$ : higher rank case, 17 pages. 2001.

\#542 T. Nakazi, The Nevanlinna counting functions for Rudin's orthogonal functions, 7 pages. 2001.

\#543 K. Sugano, On H-separable extensions of QF-3 rings, 7pages. 2001.

\#544 A. Arai, Non-relativistic limit of a Dirac-Maxwell operator in relativistic quantum electrodynamics, 27 pages. 2001.

\#545 O. Sawada, On time-local solvability of the Navier-Stokes equations in Besov spaces, 30 pages. 2001. 


\title{
DYNAMIC BOUNDARY CONDITIONS FOR HAMILTON-JACOBI EQUATIONS
}

\author{
C. M. ELLIOTT* ${ }^{*}$ Y. GIGA ${ }^{\dagger}, \Lambda N D$ S. GOTO ${ }^{\ddagger}$
}

\begin{abstract}
A non standard dynamic boundary condition for a Hamilton-Jacobi equation in one space dimension is studied in the context of viscosity solutions. A comparison principle and, hence, uniqueness is proved by consideration of an equivalent notion of viscosity solution for an altemative formulation of the boundary condition. The relationship with a Neumann condition is established. Global existence is obtained by consideration of a related parabolic approximation with a dynamic boundary condition. The problem is motivated by applications in superconductivity and interface cvolution.
\end{abstract}

1. Introduction. We consider the first order equation

$$
u_{l}-F\left(u_{x}^{2}+\gamma^{2}\right)^{1 / 2}=0 \quad \text { in } \Omega \times(0, \infty)
$$

supplemented with the dynamic boundary condition

$$
u_{\ell}-F \alpha=0 \quad \text { on } \partial \Omega \times(0, \infty),
$$

where $\Omega$ is a bounded open interval. The function $F$ and $\alpha$ are given continuous functions on $\bar{\Omega} \times[0, \infty), \partial \Omega \times[0, \infty)$ respectively and $\gamma \geq 0$ is a constant.

There are at least two sources of this problem. Consider the mean field vortex density model in a cylinder $D \times \mathbb{R}\left(D \subset \mathbb{R}^{2}\right)$ when the magnetic field $\vec{H}$ is orthogonal to the axis of the cylinder; Chapman [3]. The vorticity ficld $\vec{\omega}=\left(\nabla^{\perp} \psi, 0\right), \nabla^{\perp}=\left(-\partial_{x_{2}}, \partial_{x_{1}}\right)$ is required to satisfy the conservation of vorticity

$$
\vec{\omega}_{t}+\operatorname{curl}(\vec{\omega} \times \vec{v})=0 .
$$

*School of Mathematical Sciences, University of Sussex, Falmer, Brighton BN1 9QH, Great Britain (C.M.Elliott@sussex.ac.uk).

${ }^{\dagger}$ Department of Mathematics, Hokkaido Lniversity, Sapporo 060-0810, Japan. (giga@ math.sci.hokudai.ac.jp) The work of this author was partially supported by the Grantin-Aid for Scientific Research 10304010, 12874024, the Japan Society of the Promotion of Science.

${ }^{\ddagger}$ Department of Computational Science, Faculty of Science, Kanazawa University, Kanazawa 920-1192, Japan. (goto@kenroku.kanazawa-u.ac.jp) The work of this author was partially supported by the Grant-in-Aid for Encouragement of Young Scientists 11740108, the Japan Society of the Promotion of Science. 
If the velocity field $\vec{v}$ is of the form

$$
\vec{v}=\operatorname{curl} \vec{H} \times \vec{\omega} /|\vec{\omega}|
$$

and $\vec{H}$ is given, then the conservation of vorticity yields

$$
\psi_{t}=|\nabla \psi| F,
$$

where $F$ is a given function. Our equation (1.1) is derived by assuming that $\partial_{x_{2}} \psi=\gamma$ is a constant on $D=\Omega \times \mathbb{R}$ if wo set $u\left(x_{1}, t\right)=\psi\left(x_{1}, x_{2}, t\right)-\gamma x_{2}$. The quantity $-\psi_{t}$ on the boundary corresponds to the flux $\vec{n} \times(\vec{\omega} \times \vec{v})$ on $\partial D \times \mathbb{R}$. The condition $\psi_{t}=F \alpha$ is considered as a special caso of assigning the value of flux and we obtain (1.1), (1.2). A full system with a different boundary condition $\vec{\omega} \cdot \vec{n}=0$ is studied by Elliott, Schätzle and Stoth [6].

Another source of the problem is a surface cvolution problem with dynamic boundary condition. Consider (1.1) with $\gamma=1$. Then (1.1) is equivalent to requiring that the upward normal velocity $V$ of the graph $\Gamma_{\iota}=\{y=u(x, t)\}$ equals $F$ i.e. $V=F$. The boundary condition (1.2) is equivalent to saying that the upward velocity $v$ of $\Gamma_{l}$ on $\partial \Omega \times \mathbb{R}$ is equal to $F \alpha$, i.c. $v=F \alpha$. In [1] S. Angenent and M. Gurtin derive a dynamic boundary condition for the mean curvature flow equation. It is of the form $v=A \cos \theta+B$ where $v$ is the nomal velocity of $\partial \Gamma_{t}$ in $\partial \Omega$ and $\theta$ is the contact angle of $\Gamma_{l}$ and $\partial \Omega ; A$ and $B$ are constants. Our boundary condition corresponds to the case $A=0$.

Our goal is to study the global-in-time solvability of (1.1), (1.2) for a given initial data. Since the problem is of first order, it is convenient to handle this problem in the realm of viscosity solutions; see e.g. G. Barles [2]. We establish the comparison principle (\$3) for (1.1) and (1.2) by deriving an equivalent definition ( $(2)$ of solutions. Although the dynamic boundary value problem is studied in [2, P.102 (4.23)], it is essentially of Jeumann type and does not include (1.2). We further prove ( $(5)$ that the solution of (1.1) and (1.2) solves the Neumann problem for (1.1) with

$$
\partial u / \partial \nu=(\operatorname{Sign} F)\left\{(\alpha-\gamma)_{+}(\alpha+\gamma)\right\}^{1 / 2}
$$

in the viscosity sense, where $\beta_{+}$denotes the positive part of $\beta$ and $\operatorname{Sign} F$ denotes the sign of $F$ i.e. $\operatorname{Sign} F= \pm 1$ if $F \gtrless 0$ and $\operatorname{Sign} F=0$ if $F=0$. It might be possible to prove the comparison principle for (1.1) with the inhomogeneous data $\partial u / \partial \nu=p(t)$ when $p$ is continuous; see J. Claisse [4]. 
However, our comparison principle for (1.1) and (1.2) still holds when $F$ changes sign in which case the Neumann data in (1.3) is discontinuous and hence it is not included in the literature. Moreover, our proof is more direct and does not use (1.3).

We also prove the global existence ( $\S 4$ ) of a solution for (1.1), (1.2) when the initial data $a$ is a Lipschitz function in $\bar{\Omega}$, by using the approximate equation

$$
u_{l}-\varepsilon u_{x x}-F\left(u_{x}^{2}+\gamma^{2}\right)^{1 / 2}=0 \quad \text { in } \Omega \times(0, \infty)
$$

with the dynamic boundary condition

$$
u_{\ell}-F \alpha+\varepsilon \partial u / \partial \nu=0 \quad \text { on } \partial \Omega \times(0, \infty),
$$

where $\varepsilon$ is a positive parameter. The dynamic boundary condition for uniformly parabolic equations is well studied, for example by Hinterman [10] and Escher [7, 8]. Their results may be applied to (1.4) and (1.5) in order to yicld at least a local solution. However, since the global existence of solutions is casy to show we give a proof for global solvability of (1.4), (1.5) in the Appendix. By the maximum principle we derive a priori bounds $(\S 4)$ for the sup norms of $u_{l}^{\varepsilon}, u_{x}^{\varepsilon}, u^{\varepsilon}$ in $\bar{\Omega} \times[0, T]$ for solutions of (1.4), (1.5) independent of $\varepsilon \in(0,1)$. This yields the solution of $(1.1),(1.2)$ as a limit. The presence of the term $\varepsilon \partial u / \partial \nu$ in (1.5) is crucial in order to obtain the a priori bound.

2. Definitions and equivalent notions of solutions. Let $\Omega$ be a bounded interval $(0, L) \subset \mathbb{R}$ and $T>0$ be a constant. For brevity we set $Q=\Omega \times(0, T), \hat{Q}=\bar{\Omega} \times(0, T)$ and their closure $\bar{Q}=\bar{\Omega} \times[0, T]$. Given a mapping $k:=k(x, t, \tau, p): \hat{Q} \times \mathbb{R} \times \mathbb{R} \rightarrow \mathbb{R}$ we recall the following definitions of viscosity sub- and supersolutions $u \in C(\hat{Q})$ for $k$.

DefinITION 2.1. A function $u$ is said to be a viscosity subsolution of $k$ (in $\hat{Q})$ provided for any $(\hat{x}, \hat{t}, \phi) \in \hat{Q} \times C^{1}(\hat{Q})$ such that

$$
(u-\phi)(\hat{x}, \hat{t})=\sup _{\hat{Q}}(u-\phi)
$$

then the inequality

$$
k(\hat{x}, \hat{t}, \tau \cdot p) \leq 0
$$

holds where $\tau=\phi_{l}(\hat{x}, \hat{t})$ and $p=\phi_{x}(\hat{x}, \hat{t})$. 
DEFINITION 2.2. A function $u$ is said to be a viscosity supersolution of $k$ (in $\hat{Q})$ provided for any $(\hat{x}, \hat{t}, \phi) \in \hat{Q} \times C^{1}(\hat{Q})$ such that

$$
(u-\phi)(\hat{x}, \hat{t})=\inf _{\hat{Q}}(u-\phi)
$$

then the inequality

$$
k(\hat{x}, \hat{t}, \tau, p) \geq 0
$$

holds where $\tau=\phi_{l}(\hat{x}, \hat{t})$ and $p=\phi_{x}(\hat{x}, \hat{t})$.

Let $F$ and $\alpha$ be given functions in $C(\bar{Q}), C(\partial \Omega \times[0, T])$ respectively and $\gamma \geq 0$ be a given constant. We use the notation, since $\partial \Omega=\{0, L\}$, that $\frac{\partial}{\partial \nu}=\nu \frac{\partial}{\partial x}$ on $\partial \Omega$ with $\nu=-1$ for $x=0$ and $\nu=+1$ for $x=L$. The initial boundary value problem is

$$
\begin{cases}u_{l}-F\left(u_{x}^{2}+\gamma^{2}\right)^{1 / 2}=0 & \text { in } Q \\ u_{l}-F \alpha=0 & \text { on } \partial \Omega \times(0, T) \\ \left.u\right|_{t=0}=a & \text { on } \Omega\end{cases}
$$

In order to formulate the definition of a viscosity solution to (2.1) we define, for $(x, t, \tau, p) \in \hat{Q} \times \mathbb{R} \times \mathbb{R}$

$$
\begin{aligned}
& E(x, t, \tau, p):=\tau-F(x, t)\left(p^{2}+\gamma^{2}\right)^{1 / 2}, \\
& F_{\min }(x, t, \tau, p):= \begin{cases}E(x, t, \tau, p) & \text { if } x \in \Omega, \\
\min \{\tau-F(x, t) \alpha(x, t), E(x, t, \tau, p)\} & \text { if } x \in \partial \Omega,\end{cases} \\
& F_{\max }(x, t, \tau, p):= \begin{cases}E(x, t, \tau, p) & \text { if } x \in \Omega, \\
\max \{\tau-F(x, t) \alpha(x, t), E(x, t, \tau, p)\} & \text { if } x \in \partial \Omega .\end{cases}
\end{aligned}
$$

DEFINITION 2.3. We say that $u \in C(\bar{Q})$ is a viscosity solution of (2.1) provided $u(x, 0)=a(x), x \in \bar{\Omega}, u$ is a viscosity subsolution for $F_{\min }$ and a viscosity supersolution for $F_{\max }$.

This is the usual notion of viscosity solution for boundary value problems (cf. [5]). We give an equivalent notion of solution by introducing, for $(x, t, \tau, p) \in \hat{Q} \times \mathbb{R} \times \mathbb{R}$

$G(x, t, \tau, p):= \begin{cases}E(x, t, \tau, p) & \text { if } \quad x \in \Omega, \\ \tau-F(x, t) \max \left\{\alpha(x, t),\left(\left([p \nu \operatorname{Sign} F]_{-}\right)^{2}+\gamma^{2}\right)^{1 / 2}\right\} & \text { if } \quad x \in \partial \Omega,\end{cases}$ 
where $f_{-}$is the negative part of $f$. Set

$$
G_{B}(x, t, \tau, p)=\tau-F(x, t) \max \left\{\alpha(x, t),\left(\left([p \nu \operatorname{Sign} F]_{-}\right)^{2}+\gamma^{2}\right)^{1 / 2}\right\} .
$$

An alternative expression for $G_{B}$ is

$$
G_{B}(x, t, \tau, p)=\tau-F(x, t)\left([(\operatorname{Sign} F) p \nu-\delta][(\operatorname{Sign} F) p \nu+\delta]_{-}+\gamma^{2}+\delta^{2}\right)^{1 / 2},
$$

where $\delta=\left(\max (\alpha, \gamma)^{2}-\gamma^{2}\right)^{1 / 2}$. This identity follows from

$$
\max \left\{\alpha,\left(\left(\eta_{-}\right)^{2}+\gamma^{2}\right)^{1 / 2}\right\}=\left((\eta-\delta)(\eta+\delta)_{-}+\gamma^{2}+\delta^{2}\right)^{1 / 2}
$$

for $\eta \in \mathbb{R}$, which is casy to prove. The main purpose of this section is to prove the following proposition:

Proposition 2.4. A function $u$ is a viscosity solution of (2.1) if and only if $u \in C(\bar{Q}), u(x, 0)=a(x), x \in \Omega$, and $u$ is both a viscosity subsolution and a viscosity supersolution for $G$.

It is sufficient to prove the following Lemmas.

Lemma 2.5. A function $u$ is a viscosity supersolutiuon for $G$ if and only if $u$ is a viscosity supersolution for $F_{\max }$.

Proof. We use the notation for $(\hat{x}, \hat{t}, \phi, \tau, p)$ introduced in Definition 2.2, and $\hat{F}=F(\hat{x}, \hat{t})$ and $\hat{\alpha}=\alpha(\hat{x}, \hat{t})$. Clearly there is nothing to prove if $\hat{x} \in \Omega$ since $G$ and $F_{\max }$ agree in $\Omega$. Futhermore without loss of generality we may assume $\hat{x}=L \in \partial \Omega$ so that $\nu=1$. We suppress the word viscosity in the proof.

If $u$ is a supersolution for $G$ then $\tau \geq \hat{F} \hat{\alpha}$ so that, trivially, $u$ is a supersolution for $F_{\max }$. Thus the proof parts are concluding the situation that $u$ is a supersolution for $F_{\max }$ and proving that this implies $u$ is a supersolution for $G$. We have that

$$
\max \left\{\tau-\hat{F} \hat{\alpha}, \tau-\hat{F}\left(p^{2}+\gamma^{2}\right)^{1 / 2}\right\} \geq 0 .
$$

We may assume that $(L, \hat{t})$, by modifying $\phi$ if necessary, is a unique minimizer of $u-\phi$. It is convenient to make the following observation.

Observation (1). The following device shifts the minimizer into the interior. Let $h \in C^{1}(0, \infty)$ be a nonincreasing function such that $h(\sigma)=0$ for all 
$\sigma \geqslant 1$, and that $h(\sigma) \rightarrow+\infty$ as $\sigma \rightarrow 0$. Set $d(x):=L-x$ and $\phi^{\varepsilon}(x, t):=$ $\phi(x, t)-\varepsilon h\left(\frac{d(x)}{\varepsilon}\right)$. Let $\left(x_{\varepsilon}, t_{\varepsilon}\right)$ be a minimum point of $u-\phi^{\varepsilon}$ on $\hat{Q}$. Since

$$
\liminf _{\varepsilon \rightarrow 0}\left(u-\phi^{\varepsilon}\right)=u-\phi
$$

on $\hat{Q}$ we see that $\left(x_{\varepsilon}, t_{\varepsilon}\right) \rightarrow(L, \hat{t})$ as $\varepsilon \rightarrow 0$. Here $\lim \inf _{*}$ is the relaxed limit as in [2], i.e.,

$\liminf _{\varepsilon \rightarrow 0} f^{\varepsilon}(x, t)=\liminf _{\varepsilon \rightarrow 0}\left\{f^{\delta}(y, s) ;|y-x|<\varepsilon,|s-t|<\varepsilon, 0<\delta<\varepsilon,(y, s) \in \hat{Q}\right\}$.

Furthermore since $h(\sigma) \rightarrow \infty$ as $\sigma \rightarrow 0$ we have that, for $\varepsilon$ sufficiently small, $x_{\varepsilon}<L$ and $x_{\varepsilon} \in \Omega$. Because $u$ is a supersolution for $F_{\max }$ this implies that

$$
\phi_{t}^{\varepsilon}\left(x_{\varepsilon}, t_{\varepsilon}\right) \geq F\left(x_{\varepsilon}, t_{\varepsilon}\right)\left(\phi_{x}^{\varepsilon}\left(x_{\varepsilon}, t_{\varepsilon}\right)^{2}+\gamma^{2}\right)^{1 / 2}
$$

which yields

$$
\phi_{t}\left(x_{\varepsilon}, t_{\varepsilon}\right) \geq F\left(x_{\varepsilon}, t_{\varepsilon}\right)\left(\left(\phi_{x}\left(x_{\varepsilon}, t_{\varepsilon}\right)+h^{\prime}\left(\frac{d\left(x_{\varepsilon}\right)}{\varepsilon}\right)\right)^{2}+\gamma^{2}\right)^{1 / 2} .
$$

Observation (2). Set for $A>0, \psi(x, t):=\phi(x, t)-A d(x)$. Clearly

$$
(u-\psi)(x, t)=(u-\phi)(x, t)+A d(x) \geq(u-\psi)(L, \hat{t})
$$

for all $(x, t) \in \hat{Q}$. Since $u$ is a supersolution for $F_{\max }$, we have

$$
\max \left\{\tau-\hat{F} \hat{\alpha}, \tau-\hat{F}\left((p+A)^{2}+\gamma^{2}\right)^{1 / 2}\right\} \geq 0 .
$$

We consider separately the case $\hat{F}>0$ and $\hat{F}<0$ since the case $\hat{F}=0$ leads to $G=F_{\max }$.

Case I: $\hat{F}>0$ Again we discuss three cases.

(i) $\gamma \geq \hat{\alpha}$ and $p \geq 0$

This is immediately treated by observation (1). Sending $\varepsilon$ to zero we have

$$
\tau \geq \hat{F} \gamma=\hat{F} \max \left\{\hat{\alpha},\left(\left([p \nu]_{-}\right)^{2}+\gamma^{2}\right)^{1 / 2}\right\}
$$

(ii) $p \nu+\delta<0$ 
Using observation (1) we set that for small $\varepsilon$, we have

$$
\phi_{x}^{\varepsilon}\left(x_{\varepsilon}, t_{\varepsilon}\right)=\phi_{x}\left(x_{\varepsilon}, t_{\varepsilon}\right)+h^{\prime}\left(\frac{d\left(x_{\varepsilon}\right)}{\varepsilon}\right) \leq \phi_{x}\left(x_{\varepsilon}, t_{\varepsilon}\right)<0
$$

so that

$$
\phi_{l}\left(x_{\varepsilon}, t_{\varepsilon}\right) \geq F\left(x_{\varepsilon}, t_{\varepsilon}\right)\left(\phi_{x}\left(x_{\varepsilon}, t_{\varepsilon}\right)^{2}+\gamma^{2}\right)^{1 / 2}
$$

and sending $\varepsilon$ to zero,

$$
\tau \geq \hat{F}\left(p^{2}+\gamma^{2}\right)^{1 / 2}=\hat{F}\left((p \nu-\delta)(p \nu+\delta)_{-}+\gamma^{2}+\delta^{2}\right)^{1 / 2} .
$$

(iii) $p \nu+\delta \geq 0, \gamma<\hat{\alpha}$

This is the remaining case. From observation (2), by choosing $A$ large we find

$$
\tau \geq \hat{F} \hat{\alpha}=\hat{F}\left((p \nu-\delta)(p \nu+\delta)_{-}+\gamma^{2}+\delta^{2}\right)^{1 / 2} .
$$

Casc II: $\hat{F}<0$

(i) $\gamma \geq \hat{\alpha}, p \geq 0$

In the case $F_{\max }=\tau-\hat{F}\left(p^{2}+\gamma^{2}\right)^{1 / 2}=G_{B}$.

(ii) $\gamma \geq \hat{\alpha}, p<0$

Use observation (2) and set $A=-p>0$ which yields $F_{\max }=\tau-\hat{F} \gamma=$ $G_{B}$.

(iii) $\gamma<\hat{\alpha}, p \leq \delta$

If $|p| \leq \delta$ then $F_{\max }=\tau-\hat{F} \hat{\alpha}=G_{B}$. If $p<-\delta$ then $G_{B}=\tau-\hat{F} \hat{\alpha}$ and using observation (2) and $A=-p$ we have $F_{\max }=\tau-\hat{F} \hat{\alpha}$.

(iv) $\gamma<\hat{\alpha}, p>\delta$

In the case $F_{\max }=\tau-\hat{F}\left(p^{2}+\gamma^{2}\right)^{1 / 2}=G_{B}$.

LEMMA 2.6. A function $u$ is a viscosity subsolution for $G$ if and only if $u$ is a viscosity subsolution for $F_{\min }$.

Proof. We use the notation for $(\hat{x}, \hat{t}, \phi, \tau, p)$ introduced in Definition 2.1. Clearly there is nothing to prove if $\hat{x} \in \Omega$ since $G$ and $F_{\min }$ agree in $\Omega$. Futhermore without loss of generality we may assume $\hat{x}=L \in \partial \Omega$ so that $\nu=1$.

If $u$ is a subsolution for $G$ then

$$
\tau \leq \hat{F} \max \left\{\hat{\alpha},\left(\left([p \nu \operatorname{Sign} \hat{F}]_{-}\right)^{2}+\gamma^{2}\right)^{1 / 2}\right\}
$$


so that either $\tau \leq \hat{F} \hat{\alpha}$ or $\tau \leq \hat{F}\left(\left([p \nu \operatorname{Sign} \hat{F}]_{-}\right)^{2}+\gamma^{2}\right)^{1 / 2}$. If $\hat{F}>0$ then this implies $\min \left\{\tau-\hat{F} \hat{\alpha}, \tau-\hat{F}\left(p^{2}+\gamma^{2}\right)^{1 / 2}\right\} \leq 0$ and $u$ is a subsolution for $F_{\min }$.

On the other hand if $\hat{F}<0$ then $\tau \leq \hat{F}\left((p \nu+\delta)(p \nu-\delta)++\gamma^{2}+\delta^{2}\right)^{1 / 2}$. If $\gamma \geq \hat{\alpha}$ then either $\tau \leq \hat{F}\left(p^{2}+\gamma^{2}\right)^{1 / 2}$ or $\tau \leq \hat{F} \gamma \leq \hat{F} \hat{\alpha}$ which implies $u$ is a subsolution for $F_{\min }$. Whenever if $\hat{\alpha}>\gamma$ then either $\tau \leq \hat{F}\left(\gamma^{2}+\delta^{2}\right)^{1 / 2}=\hat{F} \hat{\alpha}$ or $\tau \leq \hat{F}\left(p^{2}+\gamma^{2}\right)^{1 / 2}$ and again $u$ is a subsolution for $F_{\min }$.

We may suppose that $u$ is a subsolution for $F_{\min }$ so that

$$
\min \left\{\tau-\hat{F} \hat{\alpha}, \tau-\hat{F}\left(p^{2}+\gamma^{2}\right)^{1 / 2}\right\} \leq 0 .
$$

It is convenient to make the following observation.

Observation (1). The following device shifts the maximizer into the interior. Let $h \in C^{1}(0, \infty)$ be a nonincreasing function such that $h(\sigma)=0$ for all $\sigma \geq 1$, and that $h(\sigma) \rightarrow+\infty$ as $\sigma \rightarrow 0$. Set $d(x):=L-x$ and $\phi^{\varepsilon}(x, t):=$ $\phi(x, t)+\varepsilon h\left(\frac{d(x)}{\varepsilon}\right)$. Let $\left(x_{\varepsilon}, t_{\varepsilon}\right)$ be a maximum point of $u-\phi^{\varepsilon}$ on $\hat{Q}$. Since

$$
\limsup _{\varepsilon \rightarrow 0} \sin ^{*}\left(u-\phi^{\varepsilon}\right)=u-\phi
$$

on $\hat{Q}$ we see that $\left(x_{\varepsilon}, t_{\varepsilon}\right) \rightarrow(L, \hat{t})$ as $\varepsilon \rightarrow 0$. Furthermore since $h(\sigma) \rightarrow \infty$ as $\sigma \rightarrow 0$ we have that, for $\varepsilon$ sufficiently small, $x_{\varepsilon}<L$ and $x_{\varepsilon} \in \Omega$. Because $u$ is a subersolution for $F_{\text {rrin }}$ this implies that

$$
\phi_{t}^{\varepsilon}\left(x_{\varepsilon}, t_{\varepsilon}\right) \leq F\left(x_{\varepsilon}, t_{\varepsilon}\right)\left(\phi_{x}^{\varepsilon}\left(x_{\varepsilon}, t_{\varepsilon}\right)^{2}+\gamma^{2}\right)^{1 / 2}
$$

which yields

$$
\phi_{l}\left(x_{\varepsilon}, t_{\varepsilon}\right) \leq F\left(x_{\varepsilon}, t_{\varepsilon}\right)\left(\left(\phi_{x}\left(x_{\varepsilon}, t_{\varepsilon}\right)-h^{\prime}\left(\frac{d\left(x_{\varepsilon}\right)}{\varepsilon}\right)\right)^{2}+\gamma^{2}\right)^{1 / 2} .
$$

Observation (2). Set for $A>0, \psi(x, t):=\phi(x, t)+A d(x)$. Clearly

$$
(u-\psi)(x, t)=(u-\phi)(x, t)-A d(x) \leq(u-\phi)(L, \hat{t})=(u-\psi)(L, \hat{t})
$$

for all $(x, t) \in \hat{Q}$. Since $u$ is a subsolution for $F_{\min }$, we have

$$
\min \left\{\tau-\hat{F} \hat{\alpha}, \tau-\hat{F}\left((p-A)^{2}+\gamma^{2}\right)^{1 / 2}\right\} \leq 0 .
$$

We treat the case $\hat{F}>0$ and $\hat{F}<0$ separately. 
Case I: $\hat{F}>0$

(i) $\gamma \geq \hat{\alpha}$

If $p \leq 0$ then $\tau \leq \hat{F}\left(p^{2}+\gamma^{2}\right)^{1 / 2}=\hat{F} \max \left\{\hat{\alpha},\left(\left([p \nu]_{-}\right)^{2}+\gamma^{2}\right)^{1 / 2}\right\}$. If $p>0$ then we use observation (2) which yiclds with $A=p, \tau \leq \hat{F} \gamma=$ $\hat{F} \max \left\{\hat{\alpha},\left(\left([p \nu]_{-}\right)^{2}+\gamma^{2}\right)^{1 / 2}\right\}$.

(ii) $\gamma<\hat{\alpha}$

If $p \leq-\delta$ then $\tau \leq \hat{F}\left(p^{2}+\gamma^{2}\right)^{1 / 2}=\hat{F}\left((p-\delta)(p+\delta)_{-}+\gamma^{2}+\delta^{2}\right)^{1 / 2}$. If $|p|<\delta$ then $\tau \leq \hat{F} \hat{\alpha}=\hat{F} \max \left\{\hat{\alpha},\left(\left([p \nu]_{-}\right)^{2}+\gamma^{2}\right)^{1 / 2}\right\}$. If $p>\delta$ then we use obscrvation (2) which yiclds with $A=p, \tau \leq \hat{F} \gamma \leq \hat{F} \max \left\{\hat{\alpha},\left(\left([p \nu]_{-}\right)^{2}+\gamma^{2}\right)^{1 / 2}\right\}$.

Casc II: $\hat{F}<0$

(i) $\gamma \geq \hat{\alpha}$ and $p \leq 0$

This is immediately treated by observation (1). Sending $\varepsilon$ to zero in

$$
\phi_{t}\left(x_{\varepsilon}, t_{\varepsilon}\right) \leq F\left(x_{\varepsilon}, t_{\varepsilon}\right)\left(\phi_{x}^{\varepsilon}\left(x_{\varepsilon}, t_{\varepsilon}\right)^{2}+\gamma^{2}\right)^{1 / 2} \leq F\left(x_{\varepsilon}, t_{\varepsilon}\right) \gamma
$$

yields $\tau \leq \hat{F} \gamma=\hat{F} \max \left\{\hat{\alpha},\left(\left([-p \nu]_{-}\right)^{2}+\gamma^{2}\right)^{1 / 2}\right\}$.

(ii) $p \nu-\delta>0$

Using observation (1) we get that for small $\varepsilon$,

$$
\phi_{x}^{\varepsilon}\left(x_{\varepsilon}, t_{\varepsilon}\right)=\phi_{x}\left(x_{\varepsilon}, t_{\varepsilon}\right)-h^{\prime}\left(\frac{d\left(x_{\varepsilon}\right)}{\varepsilon}\right) \geq \phi_{x}\left(x_{\varepsilon}, t_{\varepsilon}\right)>0
$$

so that

$$
\phi_{t}\left(x_{\varepsilon}, t_{\varepsilon}\right) \leq F\left(x_{\varepsilon}, t_{\varepsilon}\right)\left(\phi_{x}^{\varepsilon}\left(x_{\varepsilon}, t_{\varepsilon}\right)^{2}+\gamma^{2}\right)^{1 / 2} \leq F\left(x_{\varepsilon}, t_{\varepsilon}\right)\left(\phi_{x}\left(x_{\varepsilon}, t_{\varepsilon}\right)^{2}+\gamma^{2}\right)^{1 / 2}
$$

and sending $\varepsilon \rightarrow 0$,

$$
\tau \leq \hat{F}\left(p^{2}+\gamma^{2}\right)^{1 / 2}=\hat{F}\left((p \nu+\delta)(p \nu-\delta)_{+}+\gamma^{2}+\delta^{2}\right)^{1 / 2} .
$$

(iii) $p \nu-\delta \leq 0, \gamma<\hat{\alpha}$

This is the remaining case. From observation (2), by choosing $A$ large

$$
\tau \leq \hat{F} \hat{\alpha}=\hat{F}\left((p \nu+\delta)(p \nu-\delta)_{+}+\gamma^{2}+\delta^{2}\right)^{1 / 2} .
$$

REMARK. As usual the definition of subsolutions (Definition 2.1) extends to an upper semicontinuous function $u$ on $\hat{Q}$ provided that $u$ is locally bounded on $\bar{Q}$. Similarly, a supersolution is defined for lower semicontinuous functions not only for continuous functions. Results on equivalence (Lemma 2.5, Lemma 2.6) are still valid for semicontinuous functions. 
3. Comparison principle. Let $\Omega$ and $T$ be as introduced in $\S 2$ and set $\hat{Q}=\bar{\Omega} \times(0, T)$ and $\bar{Q}=\bar{\Omega} \times[0, T]$. In $\S 2$ we defined a function $G$ by

$$
\begin{aligned}
& G(x, t, \tau, p) \\
& \quad= \begin{cases}\tau-F(x, t)\left(p^{2}+\gamma^{2}\right)^{1 / 2} & \text { if } \quad x \in \Omega, \\
\tau-F(x, t) \max \left\{\alpha(x, t),\left(\left([p \nu(x) \operatorname{Sign} F(x, t)]_{-}\right)^{2}+\gamma^{2}\right)^{1 / 2}\right\} & \text { if } \quad x \in \partial \Omega,\end{cases}
\end{aligned}
$$

where $\gamma \geq 0$ is a constant and $\nu(x)$ denotes the outer unit normal of $\partial \Omega$ (i.e., $\nu(0)=-1, \nu(L)=1)$.

Theorem 3.1. Assume that $F \in C(\bar{Q}), \alpha \in C(\partial \Omega \times[0, T])$ and

$$
|F(x, t)-F(y, t)| \leq C|x-y| \quad \text { for all }(x, t),(y, t) \in \bar{Q}
$$

holds for some constant $C>0$ independent of $t$. Let $u$ and $-v$ be bounded upper semicontinuous functions on $\bar{\Omega} \times[0, T)$. Let $u$ be a viscosity subsolution for $G=0$ in $\hat{Q}$ and $v$ be a viscosity supersolution for $G=0$ in $\hat{Q}$. If $u(\cdot, 0) \leq v(\cdot, 0)$ in $\bar{\Omega}$, then $u \leq v$ in $\hat{Q}$.

Proof. Suppose that the conclusion is false. Then there would exist a point $\left(x_{1}, t_{1}\right) \in \bar{\Omega} \times[0, T)$ such that $\mu:=u\left(x_{1}, t_{1}\right)-v\left(x_{1}, t_{1}\right)>0$. For positive parameters $\lambda, \beta, \delta$, we set

$$
\begin{aligned}
& \phi(x, t, y, s)=\lambda(x-y)^{2}+\beta(t-s)^{2}+\frac{\delta}{T-t}+\frac{\delta}{T-s} \\
& \Phi(x, t, y, s)=u(x, t)-v(y, s)-\phi(x, t, y, s)
\end{aligned}
$$

and, as preparation, study the behavior of a maximum point $(\hat{x}, \hat{t}, \hat{y}, \hat{s})$ of $\Phi$ on $\bar{Q} \times \bar{Q}$. We choose a small $\delta$ (fixed here) such that $0<\delta<\left(T-t_{1}\right) \mu / 4$. So, we have

$$
\max _{\bar{Q} \times \bar{Q}} \Phi \geq \mu / 2>0
$$

and then $0 \leq \hat{t}, \hat{s}<T$ holds uniformly for $\lambda$ and $\beta$. Let $M>0$ be an upper bound of both $u$ and $-v$. By using (3.2) we see that

$$
2 M \geq u(\hat{x}, \hat{t})-v(\hat{y}, \hat{s})>\lambda|\hat{x}-\hat{y}|^{2}+\beta|\hat{t}-\hat{s}|^{2},
$$

which leads to

$$
\lambda|\hat{x}-\hat{y}|^{2}, \beta|\hat{t}-\hat{s}|^{2} \text { are bounded, }
$$


and then $|\hat{x}-\hat{y}| \rightarrow 0($ as $\lambda \rightarrow \infty),|\hat{t}-\hat{s}| \rightarrow 0($ as $\beta \rightarrow \infty)$, i.e., by taking a subsequence, there exists $\left(x_{0}, t_{0}\right) \in \hat{Q}$ such that

$$
\hat{x}, \hat{y} \rightarrow x_{0}(\operatorname{as} \lambda \rightarrow \infty), \quad \hat{t}, \hat{s} \rightarrow t_{0}(\text { as } \beta \rightarrow \infty) .
$$

(Because of our assumption $u \leq v$ at $t=0$ the time $t_{0}>0$ so that $\hat{t}, \hat{s}>0$ for sufficiently large $\lambda$ and $\beta$.) From now on, we use the same notation after taking a subsequence. By taking a subsequence, (3.3) implies that there are $\lambda_{0}$ and $\beta_{0}$ such that

$$
\lambda|\hat{x}-\hat{y}|^{2} \rightarrow \lambda_{0}(\operatorname{as} \lambda \rightarrow \infty), \quad \beta|\hat{t}-\hat{s}|^{2} \rightarrow \beta_{0}(\operatorname{as~} \beta \rightarrow \infty) .
$$

It follows that

$$
\limsup _{\lambda \rightarrow \infty, \beta \rightarrow \infty} \max _{\bar{Q} \times \bar{Q}} \Phi \leq u\left(x_{0}, t_{0}\right)-v\left(x_{0}, t_{0}\right)-\lambda_{0}-\beta_{0}-\frac{2 \delta}{T-t_{0}} .
$$

Since the left hand side is equal to or greater than $u\left(x_{0}, t_{0}\right)-v\left(x_{0}, t_{0}\right)-$ $2 \delta /\left(T-t_{0}\right)$, we have $\lambda_{0}=\beta_{0}=0$, i.e.,

$$
\lambda|\hat{x}-\hat{y}|^{2} \rightarrow 0(\operatorname{as} \lambda \rightarrow \infty), \quad \beta|\hat{t}-\hat{s}|^{2} \rightarrow 0(\operatorname{as} \beta \rightarrow \infty) .
$$

Now, let us start the main part of the proof. Note that our classifications given below are not disjoint but cover whole cases.

Case 1: $x_{0} \in \Omega$ First, we discuss the case when $\hat{x}, \hat{y}$ converge to an interior point $x_{0}$ as $\lambda \rightarrow \infty$. We may assume that $\hat{x}, \hat{y} \in \Omega$. We use an abbreviated notation $\hat{\phi}_{t}=\phi_{t}(\hat{x}, \hat{t}, \hat{y}, \hat{s})$, etc. It follows from the definition of viscosity solutions that

$$
\hat{\phi}_{l}-F(\hat{x}, \hat{t})\left(\hat{\phi}_{x}^{2}+\gamma^{2}\right)^{1 / 2} \leq 0 \quad \text { and } \quad\left(-\hat{\phi}_{s}\right)-F(\hat{y}, \hat{s})\left(\left(-\hat{\phi}_{y}\right)^{2}+\gamma^{2}\right)^{1 / 2} \geq 0 .
$$

Subtracting the second inequality from the first one we get

$$
\begin{aligned}
0 & \geq \hat{\phi}_{t}-F(\hat{x}, \hat{t})\left(\hat{\phi}_{x}^{2}+\gamma^{2}\right)^{1 / 2}-\left(\left(-\hat{\phi}_{s}\right)-F(\hat{y}, \hat{s})\left(\left(-\hat{\phi}_{y}\right)^{2}+\gamma^{2}\right)^{1 / 2}\right) \\
& \geq \frac{2 \delta}{T^{2}}+(-F(\hat{x}, \hat{t})+F(\hat{y}, \hat{s}))\left(4 \lambda^{2}|\hat{x}-\hat{y}|^{2}+\gamma^{2}\right)^{1 / 2} .
\end{aligned}
$$

We send $\beta$ to infinity and, after that, wo send $\lambda$ to infinity. Then, by using (3.5) and the Lipschitz continuity of $F(\cdot, t)$ as in (3.1), we see the second term goes to zero. Since $\delta>0$, we get a contradiction. 
Case 2: $x_{0} \in \partial \Omega$ Next, we discuss the case when $\hat{x}, \hat{y}$ go to a boundary point $x_{0}$ as $\lambda \rightarrow \infty$. If both $\hat{x}$ and $\hat{y}$ in $\Omega$ for any large $\lambda$, we get a contradiction similarly to Case 1 . We classify the rest of Case 2 into three cases, Case $2 a, 2 b, 2 c$, depending on the limit of the convergent subsequences. We further classify Case $2 \mathrm{~b}$ and $2 \mathrm{c}$ into more subcases as follows:

\begin{tabular}{||l|l||l|l||} 
Case 2a & $\hat{x}=\hat{y}=x_{0} \in \partial \Omega$ & \multicolumn{2}{||}{} \\
\hline Case 2b & $\hat{x} \in \Omega \rightarrow x_{0}$, & Case 2b(i) & $F\left(x_{0}, t_{0}\right)>0$ \\
& $\hat{y}=x_{0} \in \partial \Omega$ & Case 2b(ii) & $F\left(x_{0}, t_{0}\right)=0$ \\
& & Case 2b(iii) & $F\left(x_{0}, t_{0}\right)<0$ \\
\hline Case 2c & $\hat{x}=x_{0} \in \partial \Omega$, & Case 2c(i) & $F\left(x_{0}, t_{0}\right)<0$ \\
& $\hat{y} \in \Omega \rightarrow x_{0}$ & Case 2c(ii) & $F\left(x_{0}, t_{0}\right)=0$ \\
& & Case 2c(iii) & $F\left(x_{0}, t_{0}\right)>0$
\end{tabular}

Since the proof for Case 2c and its subcases is symmetric to that of Case $2 b$, we do not present the proof for Case $2 \mathrm{c}$.

Case 2a: When there exists a subsequence $\lambda \rightarrow \infty$ such that $\hat{x}=\hat{y}=$ $x_{0} \in \partial \Omega$, it follows that

$$
\begin{aligned}
0 \geq & \hat{\phi}_{t}-F(\hat{x}, \hat{t}) \max \left\{\alpha(\hat{x}, \hat{t}),\left(\left(\left[\hat{\phi}_{x} \nu(\hat{x}) \operatorname{Sign} F(\hat{x}, \hat{t})\right]_{-}\right)^{2}+\gamma^{2}\right)^{1 / 2}\right\} \\
& \quad-\left(\left(-\hat{\phi}_{s}\right)-F(\hat{y}, \hat{s}) \max \left\{\alpha(\hat{y}, \hat{s}),\left(\left(\left[\left(-\hat{\phi}_{y}\right) \nu(\hat{y}) \operatorname{Sign} F(\hat{y}, \hat{s})\right]_{-}\right)^{2}+\gamma^{2}\right)^{1 / 2}\right\}\right) \\
\geq & \frac{2 \delta}{T^{2}}-F\left(x_{0}, \hat{t}\right) \max \left\{\alpha\left(x_{0}, \hat{t}\right), \gamma\right\}+F\left(x_{0}, \hat{s}\right) \max \left\{\alpha\left(x_{0}, \hat{s}\right), \gamma\right\},
\end{aligned}
$$

since $\hat{\phi}_{x}=-\hat{\phi}_{y}=0$. Since the second and third terms are continuous and since the sum of them goes to zero as $\beta \rightarrow \infty$ by (3.4), we get a contradiction.

Case 2b: When there exists a subsequence $\lambda \rightarrow \infty$ such that $\hat{x} \in \Omega$ and $\hat{y}=x_{0} \in \partial \Omega$, it follows that

$$
\begin{aligned}
& \hat{\phi}_{l}-F(\hat{x}, \hat{t})\left(\hat{\phi}_{x}^{2}+\gamma^{2}\right)^{1 / 2} \leq 0, \\
& \left(-\hat{\phi}_{s}\right)-F\left(x_{0}, \hat{s}\right) \max \left\{\alpha\left(x_{0}, \hat{s}\right),\left(\left(\left[\left(-\hat{\phi}_{y}\right) \nu\left(x_{0}\right) \operatorname{Sign} F\left(x_{0}, \hat{s}\right)\right]_{-}\right)^{2}+\gamma^{2}\right)^{1 / 2}\right\} \geq 0 .
\end{aligned}
$$

We see that $\left(-\hat{\phi}_{y}\right) \nu\left(x_{0}\right)=2 \lambda\left(\hat{x}-x_{0}\right) \nu\left(x_{0}\right)<0$. We shall classify this case into three subcases depending on the sign of $F$. 
Case 2b(i): $F\left(x_{0}, t_{0}\right)>0$ When $F\left(x_{0}, t_{0}\right)>0$, we may assume that $F\left(\overline{x_{0}, \hat{s}}\right)>0$ holds for sufficiently large $\beta$. The second inequality of (3.6) implies that

$$
\begin{aligned}
0 & \leq\left(-\hat{\phi}_{s}\right)-F\left(x_{0}, \hat{s}\right) \max \left\{\alpha\left(x_{0}, \hat{s}\right),\left(\hat{\phi}_{y}^{2}+\gamma^{2}\right)^{1 / 2}\right\} \\
& \leq\left(-\hat{\phi}_{s}\right)-F\left(x_{0}, \hat{s}\right)\left(\hat{\phi}_{y}^{2}+\gamma^{2}\right)^{1 / 2} .
\end{aligned}
$$

We get a contradiction similarly to Case 1 .

Case 2b(ii): $F\left(x_{0}, t_{0}\right)=0$ When $F\left(x_{0}, t_{0}\right)=0$, we see that both $F(\hat{x}, \hat{t})$ and $F\left(x_{0}, \hat{s}\right)$ go to zero as $\lambda, \beta \rightarrow \infty$. Then it is easy to get a contradiction from (3.6).

Case 2b(iii): $F\left(x_{0}, t_{0}\right)<0$ When $F\left(x_{0}, t_{0}\right)<0$, we may assume that

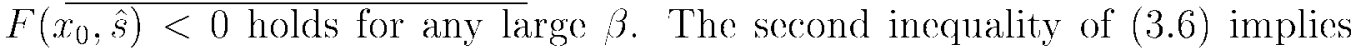
that

$$
\left(-\hat{\phi}_{s}\right)-F\left(x_{0}, \hat{s}\right) \max \left\{\alpha\left(x_{0}, \hat{s}\right), \gamma\right\} \geq 0 .
$$

If $\alpha\left(x_{0}, t_{0}\right) \leq \gamma$, sending $\beta$ to infinity; we have

$$
0 \geq \frac{2 \delta}{T^{2}}+\left(-F\left(\hat{x}, t_{0}\right)+F\left(x_{0}, t_{0}\right)\right)\left(4 \lambda^{2}\left|\hat{x}-x_{0}\right|^{2}+\gamma^{2}\right)^{1 / 2} .
$$

By using (3.j) and (3.1) the second term goes to zero as $\lambda \rightarrow \infty$. This yields a contradiction since $\delta>0$.

Case R: We must discuss the remaining case when $\alpha_{0}=\alpha\left(x_{0}, t_{0}\right)>\gamma$ from Case $2 b$ (iii) (similarly from Case 2c(iii)). Let us change $\phi$ to a new test function $\psi$ as follows:

$$
\begin{aligned}
\psi(x, t, y, s)=\lambda & \left(x-y+\operatorname{Sign} F\left(x_{0}, t_{0}\right) \frac{\left(\alpha_{0}^{2}-\gamma^{2}\right)^{1 / 2} \nu\left(x_{0}\right)}{2 \lambda}\right)^{2}+\beta(t-s)^{2} \\
& +\frac{\delta}{T-t}+\frac{\delta}{T-s} \\
=\lambda & \left(x-y-\frac{\left(\alpha_{0}^{2}-\gamma^{2}\right)^{1 / 2} \nu\left(x_{0}\right)}{2 \lambda}\right)^{2}+\beta(t-s)^{2}+\frac{\delta}{T-t}+\frac{\delta}{T-s} .
\end{aligned}
$$

We then consider the maximum point $(\tilde{x}, \tilde{t}, \tilde{y}, \tilde{s})$ of

$$
\Psi(x, t, y, s)=u(x, t)-v(y, s)-\psi(x, t, y, s)
$$


on $\bar{Q} \times \bar{Q}$. It is easy to see that $0<\tilde{t}, \tilde{s}<T$ holds uniformly for $\lambda$ and $\beta$, $|\tilde{x}-\tilde{y}| \rightarrow 0($ as $\lambda \rightarrow \infty)$ and $|\tilde{t}-\tilde{s}| \rightarrow 0($ as $\beta \rightarrow \infty)$. We claim that

$$
\tilde{x}, \tilde{y} \rightarrow x_{0}(\operatorname{as} \lambda \rightarrow \infty), \quad \tilde{t}, \tilde{s} \rightarrow t_{0}(\operatorname{as} \beta \rightarrow \infty) .
$$

If (3.7) does not hold, we see that, for some closed neighborhood $K$ of $\left(x_{0}, t_{0}\right)$, there exists $\mu^{\prime}>0$ such that

$$
\Psi(\tilde{x}, \tilde{t}, \tilde{y}, \tilde{s})-\max _{K \times K} \Psi \geq \mu^{\prime}
$$

Since $\psi$ is almost equal to $\phi$ as $\lambda \rightarrow \infty$ and $|x-y| \rightarrow 0$, we see that

$$
\Phi(\tilde{x}, \tilde{t}, \tilde{y}, \tilde{s})-\max _{K \times K} \Phi=\Phi(\tilde{x}, \tilde{t}, \tilde{y}, \tilde{s})-\Phi(\hat{x}, \hat{t}, \hat{y}, \hat{s}) \geq \mu^{\prime} / 2
$$

for large $\lambda$, which is inconsistent with the property that $(\hat{x}, \hat{t}, \hat{y}, \hat{s})$ is a global maximum point of $\Phi$. Thus (3.7) holds.

In the case when $\tilde{x}, \tilde{y} \in \Omega$ or $\tilde{x}, \tilde{y} \in \partial \Omega$ for large $\lambda$ we can discuss similarly to Case 1 and Case 2a, and obtain a contradiction, respectively. We thus study the remaining two cases, Case Rb, Re.

Case Rb: When there exists a subsequence $\lambda \rightarrow \infty$ such that $\tilde{x} \in \Omega$ and $\tilde{y}=x_{0} \in \partial \Omega$, it follows that

$$
\begin{aligned}
& \tilde{\psi}_{t}-F(\tilde{x}, \tilde{t})\left(\tilde{\psi}_{x}^{2}+\gamma^{2}\right)^{1 / 2} \leq 0, \\
& \left(-\tilde{\psi}_{s}\right)-F\left(x_{0}, \tilde{s}\right) \max \left\{\alpha\left(x_{0}, \tilde{s}\right),\left(\left(\left[\left(-\tilde{\psi}_{y}\right) \nu\left(x_{0}\right) \operatorname{Sign} F\left(x_{0}, \tilde{s}\right)\right]_{-}\right)^{2}+\gamma^{2}\right)^{1 / 2}\right\} \geq 0 .
\end{aligned}
$$

By the definition of $\psi$ we see that $\left(-\tilde{\psi}_{y}\right) \nu\left(x_{0}\right)=2 \lambda\left(\tilde{x}-x_{0}\right) \nu\left(x_{0}\right)-\left(\alpha_{0}^{2}-\right.$ $\left.\gamma^{2}\right)^{1 / 2}<0$. In the remaining case from Case $2 b$ (iii) we see that $F\left(x_{0}, t_{0}\right)<0$, and then $F\left(x_{0}, \tilde{s}\right)<0$ for large $\beta$. Thus the second inequality of (3.8) implies that

$$
\left(-\tilde{\psi}_{s}\right)-F\left(x_{0}, \tilde{s}\right) \max \left\{\alpha\left(x_{0}, \tilde{s}\right), \gamma\right\} \geq 0 .
$$

By the definition of $\psi$ we also see that $(\gamma<) \alpha_{0} \leq\left(\tilde{\psi}_{x}^{2}+\gamma^{2}\right)^{1 / 2}$. Subtracting (3.9) from the first inequality of (3.8) and sending $\beta \rightarrow \infty$ we have

$$
0 \geq \frac{2 \delta}{T^{2}}+\left(-F\left(\tilde{x}, t_{0}\right)+F\left(x_{0}, t_{0}\right)\right) \alpha_{0}
$$


since $F\left(\tilde{x}, t_{0}\right)<0$ for large $\lambda$. By (3.7) and (3.1) the second term goes to zero as $\lambda \rightarrow \infty$. This yields a contradiction since $\delta>0$.

Case Re: When there exists a subsequence $\lambda \rightarrow \infty$ such that $\tilde{x}=x_{0} \in$ $\partial \Omega$ and $\tilde{y} \in \Omega$, it follows that

$$
\begin{aligned}
& \tilde{\psi}_{t}-F\left(x_{0}, \tilde{t}\right) \max \left\{\alpha\left(x_{0}, \tilde{t}\right),\left(\left(\left[\tilde{\psi}_{x} \nu\left(x_{0}\right) \operatorname{Sign} F\left(x_{0}, \tilde{t}\right)\right]_{-}\right)^{2}+\gamma^{2}\right)^{1 / 2}\right\} \leq 0, \\
& \left(-\tilde{\psi}_{s}\right)-F(\tilde{y}, \tilde{s})\left(\left(-\tilde{\psi}_{y}\right)^{2}+\gamma^{2}\right)^{1 / 2} \geq 0 .
\end{aligned}
$$

If $\tilde{\psi}_{x} \nu\left(x_{0}\right) \geq 0$, the first inequality implies

$$
\tilde{\psi}_{l}-F\left(x_{0}, \tilde{t}\right)\left(\tilde{\psi}_{x}^{2}+\gamma^{2}\right)^{1 / 2} \leq 0
$$

since $F\left(x_{0}, \tilde{t}\right)<0$ for large $\beta$. We thus obtain a contradiction similarly to Case 1. If $\tilde{\psi}_{x} \nu\left(x_{0}\right)<0$, we have

$$
0 \leq 2 \lambda\left(x_{0}-\tilde{y}\right) \nu\left(x_{0}\right)<\left(\alpha_{0}-\gamma^{2}\right)^{1 / 2},
$$

and then $\left(\left(-\tilde{\psi}_{y}\right)^{2}+\gamma^{2}\right)^{1 / 2}<\alpha_{0}$. Since $F(\tilde{y}, \tilde{s})<0$ for large $\lambda$, $\beta$, we see that

$$
0 \geq \frac{2 \delta}{T^{2}}-F\left(x_{0}, \tilde{t}\right) \alpha\left(x_{0}, \tilde{t}\right)+F(\tilde{y}, \tilde{s}) \alpha\left(x_{0}, t_{0}\right) .
$$

We get a contradiction by the continuity of the second and third terms. The proof of Theorem 3.1 is now complete.

4. Existence theorem. Let $\Omega$ and $T$ be introduced in $\S 2$. Our goal is to show the existence of viscosity solutions of the dynamic boundary problem

$$
\left\{\begin{array}{lll}
u_{t}-F\left(u_{x}^{2}+\gamma^{2}\right)^{1 / 2}=0 & \text { in } \quad Q=\Omega \times(0, T), \\
u_{\iota}-F \alpha=0 & \text { on } \partial \Omega \times(0, T), \\
\left.u\right|_{\ell=0}=a & \text { on } \Omega .
\end{array}\right.
$$

Theorem 4.1. Assume that $F \in C^{1}(\bar{Q})$ and $\alpha \in C(\partial \Omega \times[0, T])$. Assume that a is a Lipschitz function over $\bar{\Omega}$. Then there exists a function $u \in C(\bar{Q})$ which is a unique viscosity solution of (4.1). Moreover, $\left|u_{x}\right|$ is bounded in $\bar{Q}$.

Let $\varepsilon>0$. First, we shall prove a priori estimates for a classical solution $u^{\varepsilon}$ for the approximate problem

$$
\begin{cases}u_{t}^{\varepsilon}-\varepsilon u_{x x}^{\varepsilon}=F\left(\left(u_{x}^{\varepsilon}\right)^{2}+\gamma^{2}\right)^{1 / 2} & \text { in } Q, \\ u_{l}^{\varepsilon}+\varepsilon \nu u_{x}^{\varepsilon}=F \max \left\{\alpha,\left(\left(\left[\nu u_{x}^{\varepsilon} \operatorname{Sign} F\right]_{-}\right)^{2}+\gamma^{2}\right)^{1 / 2}\right\} & \text { on } \partial \Omega \times(0, T), \\ \left.u^{\varepsilon}\right|_{t=0}=a^{\varepsilon} & \text { on } \Omega,\end{cases}
$$


where $\nu$ denotes the outer unit normal of $\partial \Omega$.

Proposition 4.2. Assume that $F \in C^{1}(\bar{Q})$ and $\alpha \in C(\partial \Omega \times[0, T])$. Assume that $a^{\varepsilon}$ is a $C^{2}$ function over $\bar{\Omega}$ and $\varepsilon a_{x x}^{\varepsilon}$ is bounded on $\bar{\Omega}$ uniformly for $\varepsilon$. Let $u^{\varepsilon}$ be a smooth solution of (4.2). Then the estimate holds

$$
\max _{\bar{Q}}\left(\left|u^{\varepsilon}\right|+\left|u_{x}^{E}\right|+\left|u_{l}^{\varepsilon}\right|\right) \leq C
$$

with some constant $C>0$ depending only on $T, \gamma,\left|a^{\varepsilon}\right|_{C^{1}(\bar{\Omega})},\left|\varepsilon a_{x x}^{\varepsilon}\right|_{C(\bar{\Omega})}$, $|F|_{C^{1}(\bar{Q})}$ and $|\alpha|_{C(\partial \Omega \times[0, T])}$.

Proof. We shall prove (4.3) by using maximum principles. (i) the estimate for $u^{\varepsilon}$ : We set $w(x, t)=e^{-t} u^{\varepsilon}(x, t)$. It follows from (4.2) that

$$
\begin{cases}w_{t}+w-\varepsilon w_{x x}=e^{-l} F\left(\left(e^{t} w_{x}\right)^{2}+\gamma^{2}\right)^{1 / 2} & \text { in } \quad Q, \\ w_{t}+w+\varepsilon \nu w_{x} & \\ =e^{-t} F \max \left\{\alpha,\left(\left(\left[e^{t} \nu w_{x} \operatorname{Sign} F\right]_{-}\right)^{2}+\gamma^{2}\right)^{1 / 2}\right\} & \text { on } \quad \partial \Omega \times(0, T), \\ w(x, 0)=a^{\varepsilon}(x) & \text { for } x \in \Omega .\end{cases}
$$

Assume that $w$ has a positive maximum in $\bar{Q}$. Let $(\hat{x}, \hat{t})$ be the maximum point of $w$ and $\lambda$ be its maximum value, i.c., $\max _{\bar{Q}} w=w(\hat{x}, \hat{t})$ and $\lambda=$ $w(\hat{x}, \hat{t})$. We assume that

$$
\lambda>\max _{\bar{\Omega}}\left|a^{\varepsilon}\right| \quad \text { and } \quad \lambda>\max _{\bar{Q}}|F| \max \left\{\max _{\partial \Omega \times[0, T]}|\alpha|, \gamma\right\}
$$

and shall show that it is inconsistent with (4.4) for $w$. First, we observe that $\hat{t}>0$ by the first inequality of $(4.5)$ and obtain $w_{t}(\hat{x}, \hat{t}) \geq 0$. When $\hat{x} \in \partial \Omega$, we also observe that $\nu(\hat{x}) w_{x}(\hat{x}, \hat{t}) \geq 0$. If $F(\hat{x}, \hat{t})>0$, then it follows from the second identity of (4.4) that

$$
\lambda \leq e^{-\hat{t}} F(\hat{x}, \hat{t}) \max \{\alpha(\hat{x}, \hat{t}), \gamma\},
$$

which is a contradiction to the second inequality of (4.5). On the other hand, $F(\hat{x}, \hat{t}) \leq 0$ implies that $\lambda \leq 0$, but it is also a contradiction. When $\hat{x} \in \Omega$, we sec that $w_{x}(\hat{x}, \hat{t})=0, w_{x x}(\hat{x}, \hat{t}) \leq 0$ to get $\lambda \leq e^{-\hat{t}} F(\hat{x}, \hat{t}) \gamma$ by the first identity of (4.4), which is a contradiction to the second incquality of (4.5). Thus we have an a priori estimate

$$
\max _{\bar{Q}} u^{\varepsilon} \leq e^{T} \max \left\{\max _{\bar{\Omega}}\left|a^{\varepsilon}\right|, \max _{\bar{Q}}|F| \max \left\{\max _{\partial \Omega \times[0, T]}|\alpha|, \gamma\right\}\right\} .
$$


We argue in the same way for a negative minimum to get

$$
\max _{\bar{Q}}\left|u^{\varepsilon}\right| \leq e^{T} \max \left\{\max _{\bar{\Omega}}\left|a^{\varepsilon}\right|, \max _{\bar{Q}}|F| \max \left\{\max _{\partial \Omega \times[0, T]}|\alpha|, \gamma\right\}\right\} .
$$

(ii) the estimate for $u_{x}^{\varepsilon}$ : We set $w(x, t)=e^{-K t} u_{x}^{\varepsilon}$ and $K=2 \max _{\bar{Q}}\left|F_{x}\right|$. It follows from (4.2) that

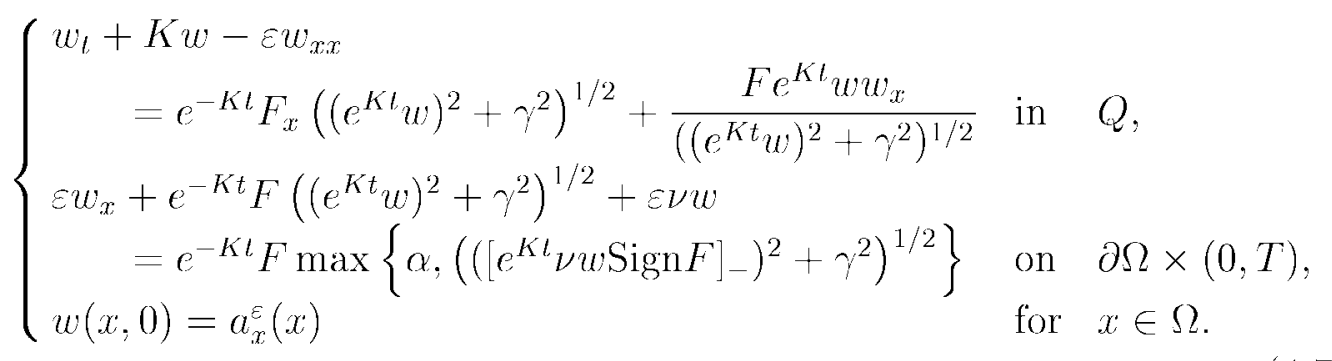

As before we take $(\hat{x}, \hat{t}), \lambda$ satisfying $\max _{\bar{Q}} w=w(\hat{x}, \hat{t})$ and $\lambda=w(\hat{x}, \hat{t})$. We assume that

$$
\lambda>\max _{\bar{\Omega}}\left|a_{x}^{\varepsilon}\right|, \quad \lambda>\max _{\partial \Omega \times[0, T]}|\alpha| \text { and } \lambda>\gamma
$$

and shall show that it is inconsistent with (4.7) for $w$. First, we see that $\hat{t}>0$ by the first inequality of (4.8) and observe $w_{l}(\hat{x}, \hat{t}) \geq 0$. When $\hat{x} \in \partial \Omega$, we also see that $\nu(\hat{x}) w_{x}(\hat{x}, \hat{t}) \geq 0$. If $\nu(\hat{x}) F(\hat{x}, \hat{t})>0$, then it follows from the second identity of $(4.7)$ after multiplying $\nu(\hat{x})$ to the both sides that

$$
\nu(\hat{x}) e^{-K \hat{\imath}} F(\hat{x}, \hat{t})\left(\left(e^{K \hat{\imath}} \lambda\right)^{2}+\gamma^{2}\right)^{1 / 2}+\varepsilon \lambda \leq \nu(\hat{x}) e^{-K \hat{\imath}} F(\hat{x}, \hat{t}) \max \{\alpha(\hat{x}, \hat{t}), \gamma\} .
$$

Dividing the both sides by $\nu(\hat{x}) e^{-K \hat{t}} F(\hat{x}, \hat{t})>0$, we obtain

$$
\left(\left(e^{K \hat{t}} \lambda\right)^{2}+\gamma^{2}\right)^{1 / 2}+\frac{\varepsilon \lambda}{\nu(\hat{x}) e^{-K \hat{\imath}} F(\hat{x}, \hat{t})} \leq \max \{\alpha(\hat{x}, \hat{t}), \gamma\} .
$$

The left hand side is strictly greater than $\lambda$, which is a contradiction to the second and third incqualities of (4.8). If $\nu(\hat{x}) F(\hat{x}, \hat{t})<0$, then a similar calculation shows that

$$
\begin{aligned}
& \nu(\hat{x}) e^{-K \hat{t}} F(\hat{x}, \hat{t})\left(\left(e^{K \hat{t}} \lambda\right)^{2}+\gamma^{2}\right)^{1 / 2}+\varepsilon \lambda \\
& \leq \nu(\hat{x}) e^{-K \hat{\imath}} F(\hat{x}, \hat{t}) \max \left\{\alpha(\hat{x}, \hat{t}),\left(\left(e^{K \hat{\imath}} \lambda\right)^{2}+\gamma^{2}\right)^{1 / 2}\right\}
\end{aligned}
$$


and, since $\nu(\hat{x}) e^{-K \hat{t}} F(\hat{x}, \hat{t})<0$,

$$
\left(\left(e^{K \hat{t}} \lambda\right)^{2}+\gamma^{2}\right)^{1 / 2}+\frac{\varepsilon \lambda}{\nu(\hat{x}) e^{-K \hat{\imath}} F(\hat{x}, \hat{t})} \geq \max \left\{\alpha(\hat{x}, \hat{t}),\left(\left(e^{K \hat{t}} \lambda\right)^{2}+\gamma^{2}\right)^{1 / 2}\right\} .
$$

Since the second term of the left hand side is negative, we get a contradiction. The term $\varepsilon \nu u_{x}$ plays a crucial role to get a contradiction for the case $\nu(\hat{x}) F(\hat{x}, \hat{t}) \neq 0$. If $F(\hat{x}, \hat{t})=0$, then the second identity of $(4.7)$ implies $\lambda \leq 0$, which is also a contradiction. When $\hat{x} \in \Omega$, we see that $w_{x}(\hat{x}, \hat{t})=0$, $w_{x x}(\hat{x}, \hat{t}) \leq 0$ and then

$$
K \lambda \leq e^{-K \hat{\imath}} F_{x}(\hat{x}, \hat{t})\left(\left(e^{K \hat{\imath}} \lambda\right)^{2}+\gamma^{2}\right)^{1 / 2}
$$

from the first identity of (4.7). The right hand side is less than or equal to $K\left(\lambda^{2}+\gamma^{2}\right)^{1 / 2} / 2$, which is a contradiction to the third inequality of (4.8). Hence, we have the estimate

$$
\max _{\bar{Q}}\left|u_{x}^{\varepsilon}\right| \leq e^{K T} \max \left\{\max _{\bar{\Omega}}\left|a_{x}^{\varepsilon}\right|, \max _{\partial \Omega \times\left[0, T^{\prime}\right]}|\alpha|, \gamma\right\}, \quad K=2 \max _{\bar{Q}}\left|F_{x}\right| .
$$

(iii) the estimate for $u_{t}^{\varepsilon}$ : We set $w(x, t)=e^{-\mu t} u_{l}^{\varepsilon}(x, t)(\mu=1)$. It follows from (4.2) that

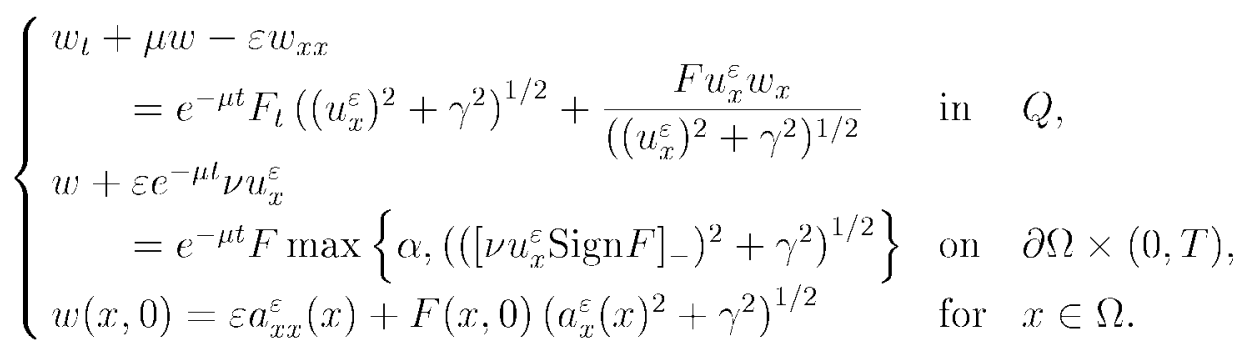

Let $(\hat{x}, \hat{t})$ and $\lambda$ satisfy $\max _{\bar{Q}} w=w(\hat{x}, \hat{t})$ and $\lambda=w(\hat{x}, \hat{t})$. We assume that

$$
\lambda>\Gamma_{0}, \quad \lambda>\Gamma_{1} \text { and } \lambda>\Gamma_{2},
$$

with

$$
\begin{aligned}
& \Gamma_{0}=\varepsilon \max _{\bar{\Omega}}\left|a_{x x}^{\varepsilon}\right|+\max _{\bar{Q}}|F|\left(\max _{\bar{\Omega}}\left|a_{x}^{\varepsilon}\right|+\gamma\right), \\
& \Gamma_{1}=\max _{\bar{Q}}|F| \max \left\{\max _{\partial \Omega \times[0, T]}|\alpha|, \max _{\bar{Q}}\left|u_{x}^{\varepsilon}\right|+\gamma\right\}, \\
& \Gamma_{2}=\max _{\bar{Q}}\left|F_{l}\right|\left(\max _{\bar{Q}}\left|u_{x}^{\varepsilon}\right|+\gamma\right) .
\end{aligned}
$$


We shall show that it is inconsistent with $(4.10)$ for $w$. First, we see that $\hat{t}>0$ by the first inequality of (4.11) and obtain $w_{l}(\hat{x}, \hat{t}) \geq 0$. When $\hat{x} \in \partial \Omega$, by the second identity of (4.10) we see that

$$
\lambda+\varepsilon e^{-\mu \hat{l}} \nu(\hat{x}) u_{x}^{\varepsilon}(\hat{x}, \hat{t}) \leq e^{-\mu \hat{l}} F(\hat{x}, \hat{t}) \max \left\{\alpha(\hat{x}, \hat{t}),\left(u_{x x}^{\varepsilon}(\hat{x}, \hat{t})^{2}+\gamma^{2}\right)^{1 / 2}\right\} .
$$

Since $u_{x}^{\varepsilon}$ is bounded uniformly for $\varepsilon$, by choosing $\varepsilon$ sufficiently small at the second term of the left hand side, we get a contradiction to the second inequality of (4.11). When $\hat{x} \in \Omega$, we sec that $w_{x}(\hat{x}, \hat{t})=0, w_{x x}(\hat{x}, \hat{t}) \leq 0$ and then

$$
\mu \lambda \leq e^{-\mu \hat{l}} F_{t}(\hat{x}, \hat{t})\left(\left(u_{x}^{\varepsilon}\right)^{2}+\gamma^{2}\right)^{1 / 2}
$$

from the first identity of (4.10), which is a contradiction to the third inequality of (4.11). Hence, we have the estimate

$$
\max _{\bar{Q}}\left|u_{t}^{\varepsilon}\right| \leq e^{T} \max \left\{\Gamma_{0}, \Gamma_{1}, \Gamma_{2}\right\}
$$

By (4.6), (4.9), (4.12) wo get the estimate (4.3) and now complete the proof of Proposition 4.2.

Remark. (1) When $F_{x} \equiv 0$, one can choose any $K>0$ in the proof (ii). So, we have

$$
\max _{\bar{Q}}\left|u_{x}^{\varepsilon}\right| \leq \max \left\{\max _{\bar{\Omega}}\left|a_{x}^{\varepsilon}\right|, \max _{\partial \Omega \times[0, T]}|\alpha|, \gamma\right\}
$$

(2) When $F_{t} \equiv 0$, we can choose any $\mu>0$ in the proof (iii). So, we have

$$
\max _{\bar{Q}}\left|u_{t}^{E}\right| \leq \max \left\{\Gamma_{0}, \Gamma_{1}, \Gamma_{2}\right\} \text {. }
$$

In a similar way to prove Proposition 4.2 one is able to prove a priori estimate which is useful to prove the global existence of solutions of (4.2) (cf. Theorem 6.1).

Corollary 4.3. Assume that $F$ and $\alpha$ are in Proposition 4.2 and that $a$ is a $C^{2}$ function over $\bar{\Omega}$. Let $\sigma \in[0,1]$ and $\varepsilon>0$. Let $v$ be a solution of

$$
\begin{cases}v_{t}-\varepsilon v_{x x}=\sigma F\left(\left(v_{x}^{\varepsilon}\right)^{2}+\gamma^{2}\right)^{1 / 2} & \text { in } Q, \\ v_{t}=\sigma F \max \left\{\alpha,\left(\left(\left[\nu v_{x} \operatorname{Sign} F\right]_{-}\right)^{2}+\gamma^{2}\right)^{1 / 2}\right\}-\sigma \varepsilon \nu v_{x} & \text { on } \partial \Omega \times(0, T), \\ \left.v\right|_{\ell=0}=\sigma a & \text { on } \Omega .\end{cases}
$$


Then the estimate holds

$$
\max _{\bar{Q}}\left(|v|+\left|v_{x}\right|\right) \leq C
$$

with some constant $C>0$ independent of $\varepsilon \in(0,1)$ and $\sigma \in[0,1]$.

Proof of Theorem 4.1. For a given Lipschitz function there is a sequence $a^{\varepsilon} \in C^{2}(\bar{\Omega})$ such that $a^{\varepsilon} \rightarrow a$ uniformly and that $\left|a_{x}^{\varepsilon}\right|_{C(\bar{\Omega})}$ and $\left|\varepsilon a_{x x}^{\varepsilon}\right|_{C(\bar{\Omega})}$ arc bounded. By Theorem 6.1 there exists a unique classical solution $u^{\varepsilon}$ of (4.2).

By the estimate (4.3) uniformly for $\varepsilon$, the Arzela-Ascoli theorem implies that these exists a function $u$ such that

$$
u^{\varepsilon} \rightarrow u \text { uniformly on } \bar{Q} \text {. }
$$

Finally, we show that $u$ is the viscosity solution of the original dynamic boundary problem (4.1). Since the proof for viscosity supersolutions is symmetric, we only prove that $u$ is a viscosity subsolution for $G=0$. To do this, let $\phi \in C^{2}(\bar{Q})$ be a test function and $(\hat{x}, \hat{t}) \in \hat{Q}=\bar{\Omega} \times(0, T)$ be the maximum point of $u-\phi$. We may assume that $(\hat{x}, \hat{t})$ is a strict maximum of $u-\phi$. Then there exists $\left(x_{\varepsilon}, t_{\varepsilon}\right)$ such that $\left(x_{\varepsilon}, t_{\varepsilon}\right) \rightarrow(\hat{x}, \hat{t})$ and $\sup _{\hat{Q}}\left(u^{\varepsilon}-\phi\right)=\left(u^{\varepsilon}-\phi\right)\left(x_{\varepsilon}, t_{\varepsilon}\right)$.

Case 1: When there exists a subsequence $\left\{\left(x_{\varepsilon}, t_{\varepsilon}\right) \in Q\right\}$, we see that $u_{t}^{\varepsilon}\left(x_{\varepsilon}, t_{\varepsilon}\right)=\phi_{t}\left(x_{\varepsilon}, t_{\varepsilon}\right), u_{x}^{\varepsilon}\left(x_{\varepsilon}, t_{\varepsilon}\right)=\phi_{x}\left(x_{\varepsilon}, t_{\varepsilon}\right)$ and $u_{x x}^{\varepsilon}\left(x_{\varepsilon}, t_{\varepsilon}\right) \leq \phi_{x x}\left(x_{\varepsilon}, t_{\varepsilon}\right)$. Since $u^{\varepsilon}$ satisfies the first identity of $(4.2)$ at $\left(x_{\varepsilon}, t_{\varepsilon}\right)$ as a classical solution, we get

$$
\phi_{l}\left(x_{\varepsilon}, t_{\varepsilon}\right)-\varepsilon \phi_{x x}\left(x_{\varepsilon}, t_{\varepsilon}\right)-F\left(x_{\varepsilon}, t_{\varepsilon}\right)\left(\phi_{x}\left(x_{\varepsilon}, t_{\varepsilon}\right)^{2}+\gamma^{2}\right)^{1 / 2} \leq 0 .
$$

By $\varepsilon \rightarrow 0$ we see that $u$ is a viscosity subsolution at $(\hat{x}, \hat{t})$.

Case 2: When $\hat{x} \in \partial \Omega$ and there is a subsequence $\left\{\left(x_{\varepsilon}, t_{\varepsilon}\right) \in \partial \Omega \times(0, T)\right\}$, we see that $u_{l}^{\varepsilon}\left(x_{\varepsilon}, t_{\varepsilon}\right)=\phi_{l}\left(x_{\varepsilon}, t_{\varepsilon}\right)$ and $\nu u_{x}^{\varepsilon}\left(x_{\varepsilon}, t_{\varepsilon}\right) \geq \nu \phi_{x}\left(x_{\varepsilon}, t_{\varepsilon}\right)$. Since $u^{\varepsilon}$ satisfies the second identity of $(4.2)$ at $\left(x_{\varepsilon}, t_{\varepsilon}\right)$ as a classical solution, we get

$$
\begin{aligned}
& \phi_{t}\left(x_{\varepsilon}, t_{\varepsilon}\right)+\varepsilon \nu \phi_{x}\left(x_{\varepsilon}, t_{\varepsilon}\right) \\
& \quad \leq F\left(x_{\varepsilon}, t_{\varepsilon}\right) \max \left\{\alpha\left(x_{\varepsilon}, t_{\varepsilon}\right),\left(\left(\left[\nu u_{x}^{\varepsilon}\left(x_{\varepsilon}, t_{\varepsilon}\right) \operatorname{Sign} F\left(x_{\varepsilon}, t_{\varepsilon}\right)\right]_{-}\right)^{2}+\gamma^{2}\right)^{1 / 2}\right\} .
\end{aligned}
$$

If $F(\hat{x}, \hat{t})>0$, we may assume that $F\left(x_{\varepsilon}, t_{\varepsilon}\right)>0$. We also see that $\left(\left[\nu u_{x}^{\varepsilon}\left(x_{\varepsilon}, t_{\varepsilon}\right)\right]_{-}\right)^{2} \leq \phi_{x}\left(x_{\varepsilon}, t_{\varepsilon}\right)^{2}$ and then

$$
\phi_{t}\left(x_{\varepsilon}, t_{\varepsilon}\right)+\varepsilon \nu \phi_{x}\left(x_{\varepsilon}, t_{\varepsilon}\right) \leq F\left(x_{\varepsilon}, t_{\varepsilon}\right) \max \left\{\alpha\left(x_{\varepsilon}, t_{\varepsilon}\right),\left(\phi_{x}\left(x_{\varepsilon}, t_{\varepsilon}\right)^{2}+\gamma^{2}\right)^{1 / 2}\right\} .
$$


By $\varepsilon \rightarrow 0$ it holds that $u$ satisfies either the first or second identity of (4.1) as a viscosity subsolution at $(\hat{x}, \hat{t})$. If $F(\hat{x}, \hat{t})<0$, we may assume that $F\left(x_{\varepsilon}, t_{\varepsilon}\right)<0$. It is casy to sec that

$$
\phi_{t}\left(x_{\varepsilon}, t_{\varepsilon}\right)+\varepsilon \nu \phi_{x}\left(x_{\varepsilon}, t_{\varepsilon}\right) \leq F\left(x_{\varepsilon}, t_{\varepsilon}\right) \alpha\left(x_{\varepsilon}, t_{\varepsilon}\right) .
$$

By $\varepsilon \rightarrow 0$ we get the second identity of (4.1) as a viscosity subsolution at $(\hat{x}, \hat{t})$. If $F(\hat{x}, \hat{t})=0$, the right hand side of $(4.14)$ vanishes as $\varepsilon \rightarrow 0$. We get $u_{l} \leq 0$ in the viscosity sense at $(\hat{x}, \hat{t})$. The Lipschitz continuity of $u$ in $x$ follows from (4.9).

5. Relation to other boundary conditions. We shall relate an inhomogeneous Neumann boundary value problem for

$$
u_{t}-F\left(u_{x}^{2}+\gamma^{2}\right)^{1 / 2}=0
$$

supplemented with the dynamic boundary

$$
u_{t}-F \alpha=0 \text {. }
$$

Formally, (5.1) and (5.2) yiclds

$$
F\left(u_{x}^{2}+\gamma^{2}\right)^{1 / 2}=F \alpha .
$$

If $F$ is not zero, this implies $u_{x}^{2}+\gamma^{2}=\alpha^{2}$. Thus we obtain

$$
\partial u / \partial \nu=u_{x} \nu= \pm\left(\alpha^{2}-\gamma^{2}\right)^{1 / 2}
$$

on the boundary. The Neumann data in (5.3) noeds more explanation since both its sign and its value for $\alpha^{2}<\gamma^{2}$ are unclear. We shall clarify these points and prove that a solution of (5.1), (5.2) solves an inhomogeneous Neumann problem in the viscosity sense (Theorem 5.1).

When we are asked to solve (5.1) and (5.2), we are tempted to integrate (5.2) in order to obtain the Dirichlet condition:

$$
u(x, t)=\int_{0}^{l} F(x, \tau) \alpha(x, \tau) d \tau+a(x), \quad x \in \partial \Omega .
$$

However, unfortunately, (5.1) with the Dirichlet condition (5.4) is not equivalent to $(5.1),(5.2)$. We shall give a counterexample in the last part of this section. 
TheOREM 5.1. Assume that $F$ and $\alpha$ are continuous on $\bar{Q}$ and $\partial \Omega \times$ $[0, T]$, respectively. Assume that $u$ is a viscosity subsolution (resp. supersolution) for $G$ in $\hat{Q}$. Then $u$ is a viscosity subsolution (resp. supersolution) of the Neumann problem of (5.1) in $\hat{Q}$ with

$$
\partial u / \partial \nu=\operatorname{Sign} F\left\{(\alpha-\gamma)_{+}(\alpha+\gamma)\right\}^{1 / 2} .
$$

Here $\beta_{+}$is the plus part of $\beta$ defined by $\beta_{+}=\max (\beta, 0)$.

Proof. We suppress the word viscosity in the proof. Since the proof for supersolutions is symmetric, we shall only present the proof for subsolutions. We may assume that $u$ is upper semicontinuous in $\hat{Q}$. Assume that $u$ is a subsolution for $G$ in $\hat{Q}$. Assume that $u-\phi$ takes its maximum over $\hat{Q}$ at $(\hat{x}, \hat{t})$ with $\hat{x} \in \bar{\Omega}, \hat{t} \in(0, T)$ for $\phi \in C^{1}(\hat{Q})$. We may assume that $\hat{x} \in \partial \Omega$ since the equation is the same in $\Omega \times(0, T)$. To simplify notation we set

$$
\tau=\phi_{t}(\hat{x}, \hat{t}), \quad p=\phi_{x}(\hat{x}, \hat{t}), \quad \hat{F}=F(\hat{x}, \hat{t}), \quad \hat{\alpha}=\alpha(\hat{x}, \hat{t}) .
$$

We have to prove that

$$
\min \left\{\tau-\hat{F}\left(p^{2}+\gamma^{2}\right)^{1 / 2}, p \nu-\operatorname{Sign} \hat{F}\left\{(\hat{\alpha}-\gamma)_{+}(\hat{\alpha}+\gamma)\right\}^{1 / 2}\right\} \leq 0
$$

To prove (5.6) we may assume that

$$
\tau-\hat{F}\left(p^{2}+\gamma^{2}\right)^{1 / 2}>0
$$

Case $1(\hat{F}<0)$. Since $u$ is a subsolution of $G=0$, we have

$$
\tau-\hat{F} \max \left\{\hat{\alpha},\left(\left([p \nu]_{+}\right)^{2}+\gamma^{2}\right)^{1 / 2}\right\} \leq 0
$$

From (5.7) and (5.8) it follows that

$$
\hat{F}\left(p^{2}+\gamma^{2}\right)^{1 / 2}<\hat{F} \max \left\{\hat{\alpha},\left(\left([p \nu]_{+}\right)^{2}+\gamma^{2}\right)^{1 / 2}\right\},
$$

or

$$
\begin{aligned}
\left(p^{2}+\gamma^{2}\right)^{1 / 2} & >\max \left\{\hat{\alpha},\left(\left([p \nu]_{+}\right)^{2}+\gamma^{2}\right)^{1 / 2}\right\} \\
& \geq\left(\left([p \nu]_{+}\right)^{2}+\gamma^{2}\right)^{1 / 2}
\end{aligned}
$$


This implies $p^{2}>\left([p \nu]_{+}\right)^{2}$ so we obtain

$$
p \nu<0
$$

Assume that $\hat{\alpha}>\gamma$. From (5.9) it follows that $\left(p^{2}+\gamma^{2}\right)^{1 / 2}>\hat{\alpha}$. This together with (5.10) implies that

$$
p \nu<-\left(\hat{\alpha}^{2}-\gamma^{2}\right)^{1 / 2} \text { for } \hat{\alpha}>\gamma
$$

By (5.10) and (5.11) wo obtain

$$
p \nu<-\left\{(\hat{\alpha}-\gamma)_{+}(\hat{\alpha}+\gamma)\right\}^{1 / 2} .
$$

We now obtain (5.6) when $\hat{F}<0$.

Case $2(\hat{F}>0)$. We note that $G=0$ is equivalent to the dynamic boundary value problem (5.1), (5.2). Since (5.7) holds, we have

$$
\tau-\hat{F} \hat{\alpha} \leq 0
$$

From (5.7) and (5.12) it follows that

$$
\hat{F}\left(p^{2}+\gamma^{2}\right)^{1 / 2}<\hat{F} \hat{\alpha}
$$

Since $\hat{F}>0$, this yields $\left(p^{2}+\gamma^{2}\right)^{1 / 2}<\hat{\alpha}$. This implies

$$
p^{2}<\hat{\alpha}^{2}-\gamma^{2} \quad \text { or } \quad|p|^{2} \leq \hat{\alpha}^{2}-\gamma^{2}=(\hat{\alpha}-\gamma)_{+}(\hat{\alpha}+\gamma) .
$$

We have thus proved (5.6) when $\hat{F}>0$.

Case $3(\hat{F}=0)$. Since $G=0$ is equivalent to the dynamic boundary value problem (5.1), (5.2), $\tau \leq 0$ is always fulfilled if $\hat{F}=0$. Thus we have proved (5.6).

We shall give a counterexample to show that the problem $(5.1),(5.2)$ is different from the Dirichlet problem (5.1), (5.4) in the viscosity sense. Wo suppress the word viscosity.

We shall give two different functions $u$ and $v$ which initially agree with each other but $u$ solves (5.1), (5.2) while $v$ solves (5.1), (5.4) when $\alpha \equiv 1, F \equiv$ $1, \alpha>\gamma$ and $\Omega=(0, \infty)$. Although it is not difficult to give such functions for $\Omega=(0, L)$ with more general $\alpha$ and $F$, we keep such assumptions to clarify 
the argument. Let $\beta$ be a constant strictly greater than $\sigma=\left(1-\gamma^{2}\right)^{1 / 2}$ so that $\eta=\left(\beta^{2}+\gamma^{2}\right)^{1 / 2}>1$. We set

$$
w(x, t)=\min \{\beta+\gamma t, \beta x+\eta t,-\sigma x+\sigma+\beta+t\}, \quad x \in \bar{\Omega} .
$$

This function is nondecreasing in $t$ and

$$
w(x, 0)=\min \{\beta x,-\sigma x+\sigma+\beta\}
$$

so that $w(x, 0)$ is lincar cxcept at $x=1$. At time $t_{0}=\beta(\eta-\gamma)^{-1}$

$$
w\left(x, t_{0}\right)=\min \left\{\beta+\gamma t_{0},-\sigma x+\sigma+\beta+t_{0}\right\} .
$$

Since $\beta \geq \sigma$, it is casy to sce that

$$
\phi_{t}-\left(\phi_{x}^{2}+\gamma^{2}\right)^{1 / 2} \leq 0 \quad \text { at }(\hat{x}, \hat{t})
$$

if $w-\phi$ attains its maximum at $(\hat{x}, \hat{t})$ over $\bar{\Omega} \times\left(0, t_{0}\right]$ even if $\hat{x} \in \partial \Omega$. So $w$ is a subsolution of $\bar{\Omega} \times\left(0, t_{0}\right]$ of $(5.1),(5.2)$ and $(5.1),(5.4)$. It is casy to sec that $w$ is a supcrsolution of (5.1), (5.2) and (5.1), (5.4) in $\bar{\Omega} \times\left(0, t_{0}\right]$ since $w_{t} \geq 1, w \geq t_{0}$ on the boundary. We now set

$$
u(x, t)=v(x, t)=w(x, t) \quad \text { for } t \leq t_{0}, x \in \bar{\Omega}
$$

and

$$
\begin{gathered}
v(x, t)=\min \{\beta+\gamma t,-\sigma x+\sigma+\beta+t\} \quad \text { for } t \geq t_{0}, x \in \bar{\Omega}, \\
u(x, t)=\max \left\{\beta+(\gamma-1) t_{0}+t-\sigma x, v(x, t)\right\} \quad \text { for } t \geq t_{0}, x \in \bar{\Omega} .
\end{gathered}
$$

As for $w$ it is easy to see that $v$ is a subsolution of both the dynamic (5.1), (5.2) and the Dirichlet problem (5.1), (5.4) in $\bar{\Omega} \times(0, \infty)$. Since $\eta>1$ so that $t_{1}=\beta(1-\gamma)^{-1}>t_{0}$, and since $v(0, t)>t$ for $t<t_{1}, v$ is a supersolution of the Dirichlet problem in $\bar{\Omega} \times\left(0, t_{1}\right)$. However, $v$ is not a supersolution in $\bar{\Omega} \times\left(0, t_{1}\right)$ of $(5.1),(5.2)$ since at the boundary $v_{t}<1$ with $v_{x}=0$.

Since $u_{t}=1$ on the boundary and since it is easy to see that $u$ is a solution of $(5.1)$ in $\Omega \times(0, \infty)$, we conclude that $u$ is a solution of $(5.1),(5.2)$ in $\bar{\Omega} \times(0, \infty)$. This is not a subsolution of $(5.1),(5.4)$ in $\bar{\Omega} \times(0, \infty)$ since $u(0, t)>t)$ by $\eta>1$ and

$$
\phi_{l}-\left(\phi_{x}^{2}+\gamma^{2}\right)^{1 / 2}>0 \quad \text { at }(0, \hat{t})
$$


if $u-\phi$ attains its maximum on $\bar{\Omega} \times(0, \infty)$ and $\hat{t}>t_{0}$. (The function $u$ is a supersolution of $(\bar{\jmath} .1),(\bar{\jmath} .4)$ since $u(0, t)>t$.) We summarize our results.

Proposition 5.2. Assume that $\alpha \equiv F \equiv 1$ and $\gamma<1$. Let $\beta>\sigma=$ $\left(1-\gamma^{2}\right)^{1 / 2}$. For $\Omega=(0, \infty)$, let $u$ and $v$ be functions defined by (5.13)(5.16). Then $u$ is a solution of the dynamic boundary problem (5.1), (5.2) in $\bar{\Omega} \times(0, \infty)$ while $v$ is a solution of the Dirichlet problem (5.1), (5.4) in $\bar{\Omega} \times\left(0, t_{1}\right)$ with $t_{1}=\beta(1-\gamma)^{-1}$. However, $u$ is not a subsolution of (5.1), (5.4) in $\bar{\Omega} \times(0, T), T>t_{0}$ while $u$ is a supersolution of (5.1), (5.4) in $\bar{\Omega} \times(0, \infty)$. The function $v$ is not a supersolution of (5.1), (5.2) while it is a subsolution of (5.1), (5.D).

\section{Appendix: Existence of solutions of approximate solutions.} Our goal is to prove:

Theorem 6.1. For $T>0$ assume that $F \in C^{1}(\bar{Q})$ and $\alpha \in C(\partial \Omega \times$ $[0, T])$ with $Q=\Omega \times(0, T)$, where $\Omega$ is a bounded open interval. Assume that $a \in C^{2}(\bar{\Omega})$ and $\gamma \in \mathbb{R}$. Then for each $\varepsilon>0$ there exists a solution $u \in C^{2,1}(Q) \cap C^{1,0}(\bar{Q})$ of

$$
\begin{cases}u_{\iota}-\varepsilon u_{x x}=F\left(u_{x}^{2}+\gamma^{2}\right)^{1 / 2} & \text { in } Q \\ u_{t}+\varepsilon \nu u_{x}=F \max \left\{\alpha,\left(\left(\left[\nu u_{x} \operatorname{Sign} F\right]_{-}\right)^{2}+\gamma^{2}\right)^{1 / 2}\right\} & \text { on } \partial \Omega \times(0, T), \\ u_{\ell=0}=a & \text { on } \Omega\end{cases}
$$

The space $C^{2,1}(Q)$ denotes the space of all $u \in C(Q)$ satisfying $u_{x}, u_{x x}$, $u_{t} \in C(Q)$. The space $X=C^{1,0}(\bar{Q})$ denotes the space of all $u \in C(\bar{Q})$ satisfying $u_{x} \in C(\bar{Q})$. The space $X$ is a Banach space equipped with the norm $\|u\|_{X}=\max \left(|u|_{C(\bar{\Omega})},\left|u_{x}\right|_{C(\bar{\Omega})}\right)$.

We shall find a solution in $X$ by a method of continuity which is a version of a fixed point argument $[9$, Theorem 11.6]. For $\sigma \in[0,1]$ we define a mapping $\mathfrak{F}_{\sigma}: X \rightarrow Y$ by

$$
\mathfrak{F}_{\sigma}(\phi)=\left(\sigma F\left(\phi_{x}^{2}+\gamma^{2}\right)^{1 / 2},\left.\sigma F \max \left\{\alpha,\left(\left(\left[\nu \phi_{x} \operatorname{Sign} F\right]_{-}\right)^{2}+\gamma^{2}\right)^{1 / 2}\right\}\right|_{\partial \Omega}-\left.\sigma \varepsilon \nu \phi_{x}\right|_{\partial \Omega}\right)
$$

where $Y=C(\bar{Q}) \times C(\partial \Omega \times[0, T])$. Let $\mathfrak{H}_{\sigma}$ denote the solution operator of the problem:

$$
\left\{\begin{array}{lll}
u_{t}-\varepsilon u_{x x}=f & \text { in } & Q \\
u_{t}=g & \text { on } & \partial \Omega \times(0, T) \\
\left.u\right|_{\iota=0}=\sigma a & \text { on } & \Omega
\end{array}\right.
$$


In other words, it is formally defined by $\mathfrak{H}_{\sigma}(f, g)=u$ for $(f, g) \in Y$. Let us give a rigorous definition. We replace the boundary condition by the standard Dirichlet condition

$$
u(x, t)=\int_{0}^{t} g(x, \tau) d \tau+\sigma a(x)=: h(x, t) .
$$

We extend $h$ linearly in $x$, i.e.

$$
\tilde{h}(x, t)=\frac{L-x}{L} h(0, t)+\frac{x}{L} h(L, t), \quad x \in \Omega,
$$

when $\Omega=(0, L)$. Then $v=u-\tilde{h}$ solves

$$
\left\{\begin{array}{lll}
v_{t}-\varepsilon v_{x x}=f-\tilde{h}_{t} & \text { in } & Q \\
v=0 & \text { on } & \partial \Omega \times(0, T), \\
\left.u\right|_{\iota=0}=\sigma a-\left.\tilde{h}\right|_{\iota=0} & \text { on } & \Omega .
\end{array}\right.
$$

Since $\tilde{h}_{l} \in C(\bar{Q})$, by the standard $L^{p}$ theory [11] there is a unique solution $v$ of the above problem and it belongs the Sobolev space $W^{2,1}(Q)$ for every $p>1$, if $a$ is cnough regular, say $a \in C^{2}(\bar{\Omega})$. The value $\mathfrak{H}_{\sigma}(f, g)$ is defined by $v+\tilde{h}$. By the construction the mapping $\mathfrak{H}_{\sigma}$ is bounded linear from $Y$ to $W_{p}^{2,1}(Q)$ for every $p>1$.

Thus the mapping $\mathfrak{H}: Y \times[0,1] \rightarrow X$ defined by $\mathfrak{H}((f, g), \sigma)=\mathfrak{H}_{\sigma}(f, g)$ is woll-defined and compact by the standard embedding theory [11]. Wo define $\mathfrak{F}: X \times[0,1] \rightarrow X$ by

$$
\mathfrak{F}(\phi, \sigma)=\mathfrak{H}\left(\mathfrak{F}_{\sigma}(\phi), \sigma\right)
$$

Evidently, $\mathfrak{F}(\phi, 0)=0$ for all $\phi \in X$. Moreover $\mathfrak{F}$ is compact since $\mathfrak{H}$ is compact and $\mathfrak{F}$ is continuous. To apply the Leray-Schauder fixed point theorem [9, Theorem 11.6] it remains to prove a priori estimate

$$
\|\phi\|_{X}<M \text { for } \phi=\mathfrak{F}(\phi, \sigma)
$$

with $M$ independent of $\phi$ and $\sigma$. Fortunately, such an estimate can be obtained as in Corollary 4.3. Note that the term $\varepsilon \nu u_{x}$ plays a crucial role, here. We have thus proved that there exists $u \in X$ such that $\mathfrak{F}(u, 1)=u$ which is the desired solution. A standard regularity theory [11] yields $u \in$ $C^{2,1}(Q)$ so Theorem 6.1 has been proved. 
Acknowledgments. This work was carried out during extremely enjoyable and stimulating visits by C. M. E. to Hokkaido University whose hospitality he gratefully acknowledges.

\section{REFERENCES}

[1] S. Angenent and M. E. Gurtin, General contact-angle conditions with and without kinetics, Quart. Appl. Math., 54 (1996), pp 557569.

[2] G. Barles, Solutions de viscosité des équations de Hamilton-Jacobi, Springer Verlag, 1994.

[3] S. J. Chapmav, A mean-field model of superconducting vortices in three dimensions, SIAM J. Appl. Math., 55 (1995), pp. 12591274.

[4] J. R. ChAIsSE, Vortex density motion in a cylindrical type II superconducter subject to a transverse applied magnetic field, 2001, D. Phil. thesis. Lniversity of Sussex.

[5] M. G. Crandall, H. Ismin and P. -L. Lions, User's guide to viscosity solutions of second order partial differential equations, Bull. Amer. Math. Soc., 27 (1992), pp. 1-67.

[6] C. M. Elliot'T, R. SCHÄTzLe And B. E. E. STOTH, Viscosity solutions of a degenerate parabolic elliptic system arising in the mean field theory of superconductivity, Arch. Rational Mech. Anal., 145 (1998), pp. 99-127.

[7] J. Escher, Quasilinear parabolic systems with dynamic boundary conditions, Comm. Partial Differential Equations, 18 (1993), pp. 13091364.

[8] J. Escher, On the qualitative behaviour of some semilinear parabolic problems, Differential Integral Equations, 8 (1995), pp. 247-267.

[9] D. Gllbarg and N. S. Tridinger, Elliptic Partial Differential Equations of Second Order, Sccond Edition, Springer Verlag, 1983.

[10] T. Hintermar, Evolution equations with dynamic boundary conditions, Proc. Roy. Soc. Edinburgh Sect. A, 113 (1989), pp. 4360.

[11] O. A. Ladyzilenskaya, V. A. Solonivikov and N. N. Ural'tseva, Lineat and Quasilinear Equations of Parabolic Type, American Mathematical Society, 1968. 